\title{
Pacific
}

Journal of

Mathematics

\section{EXCEPTIONAL DEHN SURGERY ON LARGE ARBORESCENT KNOTS}

YING-QING WU 


\title{
EXCEPTIONAL DEHN SURGERY ON LARGE ARBORESCENT KNOTS
}

\author{
YING-QING WU
}

\begin{abstract}
A Dehn surgery on a $\operatorname{knot} K$ in $S^{\mathbf{3}}$ is exceptional if it produces a reducible, toroidal or Seifert fibered manifold. It is known that a large arborescent knot admits no such surgery unless it is a type II arborescent knot. The main theorem of this paper shows that up to isotopy there are exactly three large arborescent knots admitting exceptional surgery, each of which admits exactly one exceptional surgery, producing a toroidal manifold.
\end{abstract}

\section{Introduction}

A Conway sphere for a knot $K$ in $S^{3}$ is a sphere $S$ that intersects $K$ at 4 points in such a way that the punctured sphere $S-K$ is incompressible in $S^{3}-K$. In this case the sphere $S$ cuts $\left(S^{3}, K\right)$ into two nonsplittable tangles $\left(B_{1}, \tau_{1}\right)$ and $\left(B_{2}, \tau_{2}\right)$, where $B_{i}$ is a 3-ball, and the $\tau_{i}$ are pairs of properly embedded $\operatorname{arcs}$ in $B_{i}$. An arborescent knot is obtained by gluing rational tangles together in various ways. (See, for example, [Wu 1996b] or [Gabai 1986]). An arborescent knot $K$ is large if it has a Conway sphere. It is known [Hatcher and Thurston 1985; Oertel 1984; Wu 1996b] that $K$ is large if and only if its complement is large in the sense that it contains an embedded closed essential surface.

A nontrivial Dehn surgery on a hyperbolic knot $K$ in $S^{3}$ is exceptional if the resulting manifold is either reducible, toroidal, or a small Seifert fiber space. By the Geometrization Conjecture proved by Perelman, nonexceptional surgeries yield hyperbolic manifolds. Thurston [1979] showed that a hyperbolic knot admits only finitely many exceptional surgeries.

All large arborescent knots are hyperbolic. It is known that most large arborescent knots admit no exceptional surgery. Define $T(r, s)$ to be a Montesinos tangle that is the sum of two rational tangles associated to rational numbers $r$ and $s$. (See Section 2 for more details.) A knot $K$ is an arborescent knot of type II if it has a Conway sphere cutting it into two Montesinos tangles of type $T\left(r_{i}, \frac{1}{2}\right)$. It was shown in [Wu 1996b, Theorem 3.6] that if a large arborescent knot $K$ is not of type II, then all nontrivial surgeries on $K$ are Haken and hyperbolic, so

Keywords: arborescent knots, exceptional Dehn surgery. 
there is no exceptional surgery on $K$. When $K$ is an arborescent knot of type II, all nonintegral surgeries are Haken and hyperbolic, and all integral surgeries are laminar in the sense that the resulting manifolds contain essential laminations; in particular, it is irreducible. It remains to determine which type II knots admit integer surgeries producing toroidal or Seifert fibered manifolds. The following is our main theorem, which determines all such knots and the exceptional surgeries on them. The knot $K_{1}$ in the theorem is given in Figure 2(b) on page 224. $K_{3}$ is actually the mirror image of $K_{1}$, so there are essentially only two knots up to homeomorphism of $\left(S^{3}, K\right) . K_{2}$ is obtained from $K_{1}$ by changing crossings on the right half of the diagram of $K_{1}$ in Figure 2(b).

Theorem 1.1. Let $K_{1}, K_{2}, K_{3}$ be the three knots just described (see Definition 2.3 for details). Let $K$ be a large arborescent knot in $S^{3}$, and let $\delta$ be a nonmeridional slope on $\partial N(K)$. Then $K(\delta)$ is an exceptional surgery if and only if $(K, \delta)$ is isotopic to $\left(K_{1}, 3\right),\left(K_{2}, 0\right)$ or $\left(K_{3},-3\right)$, in which case $K(\delta)$ is toroidal.

The essential punctured torus $F$ constructed in the exterior of the knots $K$ has four boundary components, and that is minimal; see Lemma 6.2 and Proposition 5.8 below. The referee pointed out that this is an interesting fact, since there are few examples of classes of knots with such a property. Some examples were given by Eudave-Muñoz [1997] and others, more recently, by Teragaito [2008]. Those were constructed via double branched covers and the punctured torus was not explicitly described in the knot exterior. The examples constructed in this paper are perhaps the simplest known knots whose exterior contains such a punctured torus $F$, and $F$ is explicitly constructed. We would like to thank the referee for this as well as some other helpful comments.

The paper is organized as follows. In Section 2 we define some special disks in rational tangle spaces. These are the pieces that will be used to build the toroidal surfaces in the exteriors of the knots in Theorem 1.1. Section 3 defines an index $i(G, Q)$ for a surface $G$ relative to $Q$, and proves its additivity and some other properties. Now let $K$ be a type II knot, which is the union of two Montesinos tangles $T_{i}=T\left(r_{i}, \frac{1}{2}\right)$. Let $F$ be a punctured essential torus in the exterior of $K$ with integer boundary slope, and let $F_{i}$ be the intersection of $F$ with the tangle space of $T_{i}$. The important fact is that $F$ can be chosen so that each component $G$ of $F_{i}$ must have zero index relative to the tube around the unknotted string of $T_{i}$. It is then shown in Section 4 that $G$ must be a special surface in the sense that it is the union of special disks in the two rational tangle spaces. This quickly leads to a proof that $r_{i} \equiv \pm \frac{1}{3} \bmod 1$. In Section 5 we define the relative framing for a surface in a tangle space. This can be used to calculate the boundary slope of the surface $F=F_{1} \cup F_{2}$, and to show that if $F$ has integer boundary slope then its intersection with the punctured Conway sphere must have a very special configuration, which 
completely determines the gluing map between the two tangles. It follows that if $K(\delta)$ is toroidal, then $K$ must be one of the three knots in the theorem, and there is only one possible choice of $F$. In Section 6 it is shown that the surface $F$ constructed is incompressible and $\partial$-incompressible, and that it gives rise to an essential torus in the surgered manifold. This, together with some known results about surgery on type II knots, will complete the proof of Theorem 1.1.

Unless otherwise stated, a surface in a 3-manifold $M$ is assumed to be either on the boundary of $M$ or properly embedded in $M$. Given a set $X$ in a surface or 3-manifold, denote by $N(X)$ a regular neighborhood of $X$, and by $|X|$ the number of components in $X$. If $P$ is a surface in a 3-manifold $M$, denote by $M \mid P$ the manifold obtained by cutting $M$ along $P$.

\section{Special disks in tangle spaces}

A tangle $T$ is a pair $(B, \tau)$, where $B$ is a 3-ball with four specified points on $\partial B$, and $\tau=\tau_{1} \cup \tau_{2}$ is a pair of arcs in $B$ connecting these points. We identify $B$ with either a pillowcase with $\partial \tau$ the four corners, or the one point compactification of the lower half-space, that is, $B=\hat{R}_{-}^{3}=\{(x, y, z) \mid z \leq 0\} \cup\{\infty\}$, with $\partial \tau$ identified to the four points $( \pm 1, \pm 1)$ and the front side of the pillowcase identified to the square $[-1,1] \times[-1,1]$ in $\hat{R}^{2}=R^{2} \cup\{\infty\}$, which is identified to $\left(R^{2} \times\{0\}\right) \cup\{\infty\}=\partial \hat{R}_{-}^{3}$.

Given a tangle $T=(B, \tau)$, let $E(T)$ be the closure of $B-N(\tau)$, called the tangle space of $T$. Let $S(T)=\partial B=\hat{R}^{2}$, and let $P(T)$ be the 4-punctured sphere $\partial B \cap E(T)$. Let $S_{+}(T)$ and $S_{-}(T)$ be the closures of the right and left half-planes of $\hat{R}^{2}=\partial B$, respectively. Similarly, define $P_{ \pm}(T)=S_{ \pm}(T) \cap E(T)$, a twice-punctured disk. Denote by $U(T)=U_{+}(T) \cup U_{-}(T)$ the two annuli $\partial E(T)-\operatorname{Int} P(T)$, with $U_{+}(T)$ the one containing the upper right component of $\partial P$ on $\hat{R}^{2}$. We now have a decomposition of the boundary of $E(T)$ :

$$
\partial E(T)=P_{-}(T) \cup P_{+}(T) \cup U_{-}(T) \cup U_{+}(T) .
$$

We refer the reader to [Conway 1970] or [Wu 1996a] for the definition of rational tangles. Roughly speaking, the strings $\tau$ of a rational tangle of slope $r=p / q$ are obtained by pushing into the interior of $B$ the interior of two arcs of slope $r$ on the boundary of the pillowcase $B$ connecting the four corners of $B$. Throughout this paper we assume that $q \geq 2$.

Given two tangles $T_{i}=\left(B_{i}, \tau_{i}\right)$, we may construct a new tangle $T_{1}+T_{2}$ by identifying the disk $S_{+}\left(T_{1}\right) \subset \hat{R}^{2}$ with $S_{-}\left(T_{2}\right) \subset \hat{R}^{2}$, using the map $(x, y) \rightarrow$ $(-x, y)$, and then identifying $B_{1} \cup B_{2}$ to $B=\hat{R}_{-}^{3}$ so that a boundary point of $B_{1}$ or $B_{2}$ on $\partial\left(B_{1} \cup B_{2}\right)$ is mapped to the point with the same coordinates on $\partial B=\hat{R}^{2}$. Denote by $T\left(r_{1}, r_{2}\right)$ the Montesinos tangle $T\left(r_{1}\right)+T\left(r_{2}\right)$. 
Two tangles $T_{1}, T_{2}$ are weakly equivalent if $T_{1}$ can be deformed to $T_{2}$ by an isotopy $\varphi_{t}$ of $B$. They are $P$-equivalent if the isotopy $\varphi_{t}$ is rel $\partial P_{+}\left(T_{1}\right)$, and equivalent if $\varphi_{t}$ is rel $S(T)$. Thus, for example, $T(r)$ is $P$-equivalent to $T(r+k)$ for any integer $k$, and $T\left(\frac{1}{3},-\frac{1}{2}\right)$ is $P$-equivalent to $T\left(\frac{1}{3}, \frac{1}{2}\right)$. Two tangles are considered the same if they are equivalent.

A surface $F$ in $E(T)$ is tight if $\partial F \neq \varnothing$, and it intersects each of $P_{ \pm}$and $U_{ \pm}$ in essential arcs or essential circles. In this case, $\partial F$ is a set of essential loops on $\partial E(T)$. Thus a tight disk is an essential disk in $E(T)$ (that is, a compressing disk of $\partial E(T)$ ), and any essential disk in $E(T)$ is isotopic to a tight disk.

The strings $\tau$ in a rational tangle $T(p / q)=(B, \tau)$ are rel $\partial \tau$ isotopic to a pair of $\operatorname{arcs} \tau^{\prime}=\tau_{1}^{\prime} \cup \tau_{2}^{\prime}$ on $\partial B$. Specifically, let $\tau_{2}^{\prime}$ be the one with an endpoint at $(1,1) \in \hat{R}^{2}$. Let $\tau_{0}^{\prime}$ be a pair of horizontal arcs on $R^{2}$ connecting $\partial \tau$. Let $c_{i}=\tau_{i}^{\prime} \cap P(T)$ for $i=0,1,2$, and let $c_{3}$ be the curve on $P$ that separates $c_{1}$ and $c_{2}$, which is unique up to isotopy. Thus, for example, for $T\left(\frac{1}{3}\right)$ the curves $c_{0}, c_{1}, c_{2}$ are shown in parts (1)-(3) of Figure 1, and for $T\left(-\frac{1}{2}\right)$ the curves $c_{1}, c_{2}, c_{3}$ are shown in parts (4)(6). A disk $D$ in $E(T)$ is a special disk if its intersection with $P(T)$ is one of these curves. It is further required that $q \leq 3$ if $D \cap P(T)=c_{1}, c_{2}$, and $q=2$ if $D \cap P(T)=c_{3}$. More explicitly, we have Definition 2.1, illustrated by Figure 1;

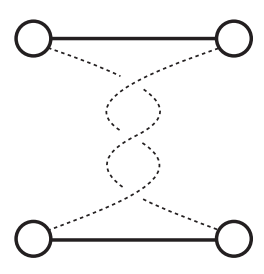

(1)

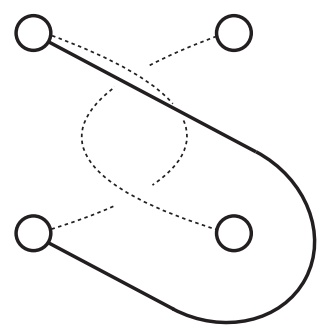

(4)

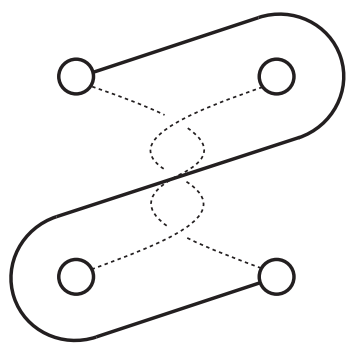

(2)

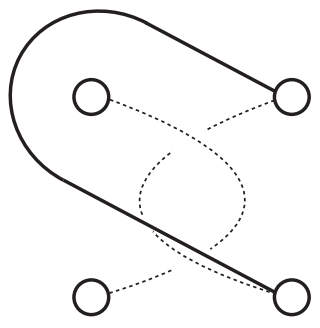

(5)

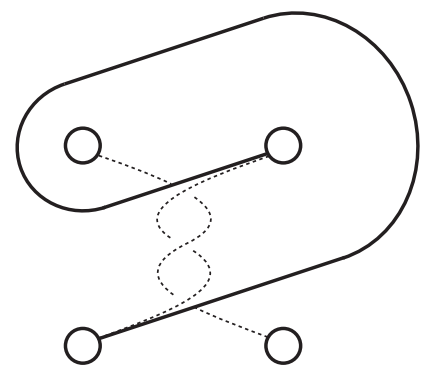

(3)

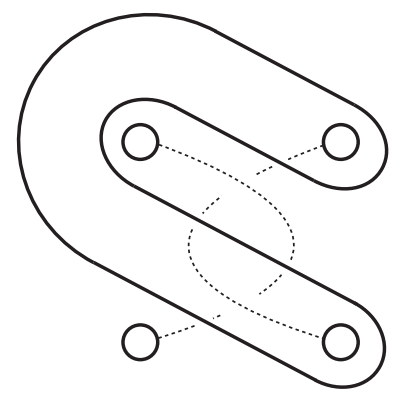

(6)

Figure 1 
part $(k)$ of the figure (with $k=1,2, \ldots 6)$ depicts the boundary curve of a type $(k)$ special disk.

Definition 2.1. Let $T=T( \pm 1 / q)$. A disk $D$ in $E(T)$ is a special disk if it is of one of the following types.

- Type (1): $T=T( \pm 1 / q), q$ odd, and $D \cap P(T)=c_{0}$.

- Type (2): $T=T\left( \pm \frac{1}{3}\right)$, and $D \cap P(T)=c_{1}$.

- Type (3): $T=T\left( \pm \frac{1}{3}\right)$, and $D \cap P(T)=c_{2}$.

- Type (4): $T=T\left( \pm \frac{1}{2}\right)$, and $D \cap P(T)=c_{1}$.

- Type (5): $T=T\left( \pm \frac{1}{2}\right)$, and $D \cap P(T)=c_{2}$.

- Type (6): $T=T\left( \pm \frac{1}{2}\right)$, and $D \cap P(T)=c_{3}$.

Lemma 2.2. Let $T=T(p / q)$ with $q=2$ or odd. Let $Q=P_{+}(T)$ if $q \geq 3$, and $Q=P_{-}(T) \cup U_{+}(T)$ if $q=2$. Suppose $D$ is a tight disk in $T(p / q)$ such that $|D \cap Q| \leq 2$. Then $T$ is $P$-equivalent to $T( \pm 1 / q)$, and $D$ is a special disk. In particular, $Q$ and $Q^{\prime}=\partial E(T)-$ Int $Q$ are incompressible, and there is no disk in $E(T)$ intersecting each of $Q$ and $Q^{\prime}$ at a single essential arc.

Proof. This is essentially [Wu 1996a, Lemmas 2.1 and 2.2]. Let $P=P_{+}(T)$ if $q \geq 3$, and $P=P_{-}(T)$ if $q=2$. Clearly $D$ must intersect $P$ in a nonempty set of arcs, since otherwise we would have $T=T\left(\frac{1}{0}\right)$. Since $|D \cap P| \leq 2, D$ is a monogon or bigon as classified in [Wu 1996a, Lemmas 2.1 and 2.2]. When it is a monogon, $D$ is a special disk of type (5). Bigons appear when $T$ is a torus tangle or wrapping tangle (see [Wu 1996a] for definition), or a twist tangle; but since $T$ is rational and $q$ equals 2 or is odd, the first two cases do not happen. Thus, from the proof of [Wu 1996a, Lemma 2.2], we see that if $D$ is a bigon, then it is one of the types (1), (2), (3), (4) or (6) in Definition 2.1, or $T$ is $P$-equivalent to $T\left(\frac{1}{4}\right)$ and $D$ intersects $P$ in two arcs with boundary on the outer component of $\partial P$, but since we have assumed that $q$ equals two or is odd, the latter case is impossible.

Denote by $T\left(r_{1}, r_{2} ; n\right)$ the tangle obtained from $T\left(r_{1}, r_{2}\right)=\left(B_{3}, \tau\right)$ by twisting the two lower endpoints of $\tau$ by $n$ left-hand half-twists. See Figure 2(a) for the tangle $T\left(\frac{1}{3},-\frac{1}{2} ; 4\right)$.

Definition 2.3. Let $\eta: \hat{R}^{2} \rightarrow \hat{R}^{2}$ be the map that is a $\pi / 2$ counterclockwise rotation about the origin followed by a reflection along the $y$-axis. Define three knots $K_{1}, K_{2}, K_{3}$ by

$$
\begin{gathered}
\left(S^{3}, K_{1}\right)=T\left(\frac{1}{3},-\frac{1}{2} ; 4\right) \cup_{\eta} T\left(\frac{1}{3},-\frac{1}{2} ; 4\right), \\
\left(S^{3}, K_{2}\right)=T\left(\frac{1}{3},-\frac{1}{2} ; 4\right) \cup_{\eta} T\left(-\frac{1}{3}, \frac{1}{2} ;-4\right), \\
\left(S^{3}, K_{3}\right)=T\left(-\frac{1}{3}, \frac{1}{2} ;-4\right) \cup_{\eta} T\left(-\frac{1}{3}, \frac{1}{2} ;-4\right) .
\end{gathered}
$$




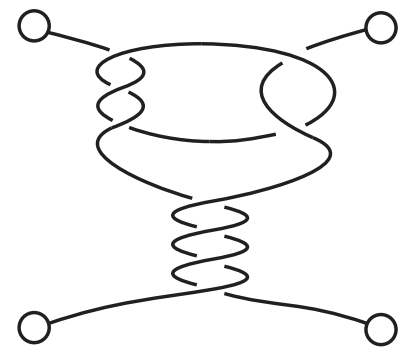

(a)

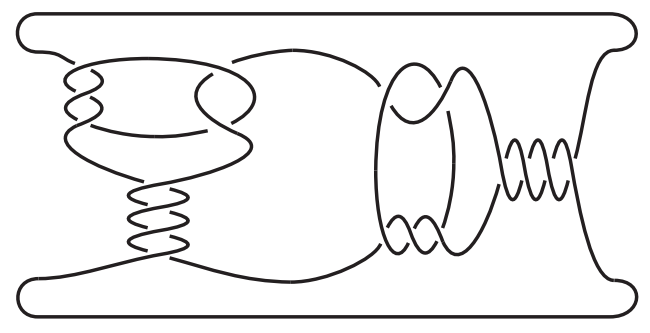

(b)

Figure 2

Alternatively, $K_{i}$ can be obtained by shifting the first tangle to the left, the second tangle to the right, rotating the second tangle counterclockwise by an angle of $\pi / 2$, and then connecting the endpoints of the tangles by $\operatorname{arcs}$ on $R^{2}$ that are horizontal except near the endpoints of the strings of the tangles, as shown in Figure 2(b) for $K_{1}$. Note that $K_{3}$ is the mirror image of $K_{1}$, and $K_{2}$ is obtained from $K_{1}$ by changing the right half of $K_{1}$ to its mirror image. Theorem 1.1 shows that these are the only large arborescent knots that admit exceptional surgery, and each of them admits exactly one such surgery.

\section{Index of essential surfaces}

Let $Q$ and $F$ be surfaces in $M$, intersecting in general position. Denote by $a(F, Q)$ the number of arc components of $F \cap Q$. The index of $F$ in $M$ relative to $Q$ is defined as

$$
i(F, Q)=\chi(F)-\frac{1}{2} a(F, Q),
$$

where $\chi(F)$ is the Euler characteristic of $F$. This is the same as the cusped Euler characteristic defined in [Wu 1998] for sutured manifolds, except that now $Q$ is not required to be "cusps", which by definition is a set of annuli and tori on the boundary of a 3-manifold.

Lemma 3.1. Let $Q$ be a surface on $\partial M$, and $Q^{\prime}$ an essential surface properly embedded in $M$ and disjoint from $Q$. Let $M^{\prime}=M \mid Q^{\prime}$, and let $Q_{1}^{\prime}, Q_{2}^{\prime}$ be the two copies of $Q^{\prime}$ on $\partial M^{\prime}$. Let $F$ be a surface in $M$, and let $F^{\prime}=F \mid Q^{\prime}$ be the corresponding surface in $M^{\prime}$. Then

$$
i\left(F^{\prime}, Q_{1}^{\prime} \cup Q_{2}^{\prime} \cup Q\right)=i(F, Q) .
$$

Proof. Put $k=a\left(F, Q^{\prime}\right)$. Note that $Q_{1}^{\prime}, Q_{2}^{\prime}, Q$ are mutually disjoint compact surfaces on $\partial M^{\prime}$, and hence $a\left(F^{\prime}, Q_{1}^{\prime} \cup Q_{2}^{\prime} \cup Q\right)=2 k+a(F, Q)$. Since $Q^{\prime}$ intersects $F$ in $k$ arcs and possibly some circle components, after cutting $F$ along $F \cap Q^{\prime}$ we 
have $\chi\left(F^{\prime}\right)=\chi(F)+k$. It follows that

$$
\begin{aligned}
i(F, Q) & =\chi(F)-\frac{1}{2} a(F, Q)=\chi\left(F^{\prime}\right)-k-\frac{1}{2} a(F, Q) \\
& =\chi\left(F^{\prime}\right)-\frac{1}{2} a\left(F^{\prime}, Q_{1}^{\prime} \cup Q_{2}^{\prime} \cup Q\right) \\
& =i\left(F^{\prime}, Q_{1}^{\prime} \cup Q_{2}^{\prime} \cup Q\right) .
\end{aligned}
$$

The lemma shows that index is invariant when cutting along a surface disjoint from $Q$. It is important to assume that $Q$ is a compact surface and is disjoint from $Q^{\prime}$, otherwise the lemma may not be true.

The most useful case is when $Q^{\prime}$ is a separating surface, cutting $M$ into $M_{1}$ and $M_{2}$. The following additivity lemma follows immediately from Lemma 3.1.

Lemma 3.2 (Additivity of index). Suppose $Q$ is a compact subsurface of $\partial M$, and $Q^{\prime}$ is a separating surface in $M$ disjoint from $Q$, cutting $M$ into $M_{1}$ and $M_{2}$. Let $Q_{i}^{\prime}$ be the copy of $Q^{\prime}$ on $\partial M_{i}$, let $Q_{i}=\left(Q \cap M_{i}\right) \cup Q_{i}^{\prime}$, and let $F_{i}=F \cap M_{i}$. Then

$$
i(F, Q)=i\left(F_{1}, Q_{1}\right)+i\left(F_{2}, Q_{2}\right) .
$$

Lemma 3.3. Let $T=T(p / q)$ with $q=2$ or odd. Let $Q=P_{+}(T)$ for $q>2$, and $Q=P_{-}(T) \cup U_{+}(T)$ for $q=2$. Let $F$ be a tight disk in $E(T)$. Then $i(F, Q) \leq 0$, and equality holds if and only if $T$ is $P$-equivalent to $T( \pm 1 / q)$ and $F$ is a special disk.

Proof. It is easy to check that each special disk in Definition 2.1 intersects $Q$ in two arcs and hence has $i(F, Q)=0$. Conversely, if $i(F, Q) \geq 0$ then $|F \cap Q| \leq 2$, so by Lemma 2.2 it is a special disk and hence $i(F, Q)=0$.

Lemma 3.4. Suppose $T=T\left(p / q, \frac{1}{2}\right)$ and $F$ is a tight surface in $E(T)$. Let $U=$ $U_{+}(T)$. Then $i(F, U) \leq 0$. In particular, $P(T)$ and $\partial E(T)-U$ are incompressible, and there is no disk in $E(T)$ intersecting $U_{+}(T)$ at a single essential arc.

Proof. If $i(F, U)>0$, then $F$ is a disk and $F \cap U$ has at most one arc component. Isotope the decomposition surface $P$ in $E(T)=E(T(p / q)) \cup_{P} E\left(T\left(\frac{1}{2}\right)\right)$ so that $F \cap P$ is minimal. Then an innermost circle/outermost arc argument would lead to a contradiction of Lemma 3.3 because one of the (at least two) disks cut off by outermost arcs will be disjoint from $U$ and hence is isotopic to a tight disk with positive index.

\section{Special surfaces in knot exterior}

Throughout this section we assume that $K$ is a type II knot that is the union of two length 2 Montesinos tangles $T_{i}=\left(B_{i}, \tau_{i}\right)=T\left(p_{i} / q_{i}, \frac{1}{2}\right)=T_{i 1}+T_{i 2}$, where $T_{i 1}=T\left(p_{i} / q_{i}\right)$ and $T_{i 2}=T\left(\frac{1}{2}\right)$.

Let $S=\partial B_{i}$, and let $P=P\left(T_{1}\right)=P\left(T_{2}\right)=S \cap E(K)$, which cuts $E(K)$ into $E\left(T_{1}\right)$ and $E\left(T_{2}\right)$. Let $P_{i}=E\left(T_{i 1}\right) \cap E\left(T_{i 2}\right)$ be the twice-punctured disk cutting 
$E\left(T_{i}\right)$ into $E\left(T_{i 1}\right)$ and $E\left(T_{i 2}\right)$. Write $T_{i j}=\left(B_{i j}, \tau_{i j}\right)$. Thus $E(K)$ is the union of four rational tangle spaces $E\left(T_{i j}\right), i, j=1,2$. Let $U_{i}=U_{+}\left(T_{i}\right)$, which is the component of $U\left(T_{i}\right)$ lying in $E\left(T_{i 2}\right)$.

Definition 4.1. A surface $F$ in $E(K)$ or $E\left(T_{i}\right)$ is a special surface if it intersects each $E\left(T_{i j}\right)$ in special disks.

The purpose of this section is to show that if $F$ is an essential punctured torus in $E(K)$ with integer slope, then it is a special surface up to isotopy. We use this result to show that $q_{i}=3$ for $i=1,2$.

If $F$ is a compact surface in $E(T)$ intersecting $U(T)$ in $\operatorname{arcs}$ on $\partial F$, then $F-$ $U(T)$ is $F$ with the $\operatorname{arcs} F \cap U(T) \subset \partial F$ removed, so it is noncompact. An arc $\beta$ in $F-U(T)$ is considered essential if it does not cut off a compact disk from $F-U(T)$. Thus, for example, if $F$ is a disk intersecting $U(T)$ in two $\operatorname{arcs}$ on $\partial F$, then there is exactly one essential arc on $F-U(T)$ up to isotopy.

A $P$-compressing disk of a surface $F$ in $E(T)$ is a disk $D$ in $E(T)$ such that $\partial D=\alpha \cup \beta$, where $\alpha$ is an arc on $P$ and $\beta=D \cap(F-U(T))$ is an arc on $F-U(T)$ that is essential. If such a disk exists, then $F$ is $P$-compressible; otherwise it is $P$-incompressible.

Lemma 4.2. Let $F$ be a punctured essential torus in $E(K)$ such that $\partial F$ has integer slope on $\partial E(K)$, and the complexity $(|F \cap \partial P|,|F \cap P|)$ is minimal in lexicographic order. Let $F_{j}$ be a component of $F \cap E\left(T_{i}\right)$. Then

(i) $F_{j}$ is tight,

(ii) $i\left(F_{j}, U_{i}\right)=0$, and

(iii) $F_{j}$ is incompressible and $P$-incompressible.

Proof. (i) Since $|F \cap \partial P|$ is minimal, $F$ intersects each of the four annuli $\partial N(K) \mid \partial P$ in essential arcs. By [Wu 1996a, Lemma 3.3], each $E\left(T_{i}\right)$ is a handlebody and hence irreducible, so if $F \cap P$ contains a trivial loop on $P$, then an innermost circle argument would show that one could isotope $F$ to reduce $|F \cap P|$ without increasing $|F \cap \partial P|$, which is a contradiction. If $F \cap P$ has a trivial $\operatorname{arc}$ on $P$, then an outermost one would cut off a $\partial$-compressing disk for $F$, which is impossible because $F$ is essential.

(ii) Since $P$ is incompressible [Wu 1996a, Lemma 3.3], each circle component of $F \cap P$ is also essential on $F$. It follows that each boundary component of $F \cap E\left(T_{i}\right)$ is a nontrivial loop on $\partial E\left(T_{i}\right)$, so each disk component of $F \cap E\left(T_{i}\right)$ is an essential disk in $E\left(T_{i}\right)$.

Let $\hat{F}$ be the torus obtained from $F$ by capping off each boundary component of $F$ with a disk. Define a graph $\Gamma$ on $\hat{F}$ with the attached disks as fat vertices and $P \cap F$ as edges. A component of $P \cap F$ is either a circle edge or an arc edge of 
$\Gamma$, depending on whether it is a circle or arc. A circle edge is not incident to any vertex, so $\Gamma$ is actually a graph in which some edges are loops without vertices.

Since $\partial F$ has integer boundary slope and $P$ has 4 boundary components, each vertex $v$ of $\Gamma$ has valence 4 . Denote by $C$ the number of corners at all vertices of $\Gamma$ that lie in either $U_{1}$ or $U_{2}$, where $U_{i}=U_{+}\left(T_{i}\right)$, which is the component of $\partial N(K) \cap E\left(T_{i}\right)$ lying in the $E\left(T_{i 2}\right)=E\left(T\left(\frac{1}{2}\right)\right)$ part. Denote by $V$ and $E$ the numbers of vertices and arc edges of $\Gamma$. Since the boundary of each vertex of $\Gamma$ travels through each of $U_{1}$ and $U_{2}$ once, we have $C=2 V$. Since each vertex is incident to 4 arc edge endpoints, we also have $E=2 V$. Let $F_{j}$ be the faces of $\Gamma$, and assume it lies in $E\left(T_{i}\right)$. The Euler characteristic formula, whose validity for graphs with circle edges one can verify, gives

$$
\begin{aligned}
0 & =\chi(\hat{F})=V-E+\sum \chi\left(F_{j}\right)=-V+\sum \chi\left(F_{j}\right)=-\frac{1}{2} C+\sum \chi\left(F_{j}\right) \\
& =\sum\left(\chi\left(F_{j}\right)-\frac{1}{2}\left|F_{j} \cap U_{i}\right|\right)=\sum i\left(F_{j}, U_{i}\right) .
\end{aligned}
$$

By (i) and Lemma 3.4, we have $i\left(F_{j}, U_{i}\right) \leq 0$ for each component $F_{j}$ of $F \cap E\left(T_{i}\right)$. Since these are exactly the faces of $\Gamma$, it follows that $i\left(F_{j}, U_{i}\right)=0$ for all $F_{j}$.

(iii) Since $F$ is incompressible and $|F \cap P|$ is minimal, it is easy to see that $F_{j}$ is also incompressible. By (ii), $F_{j}$ is either a disk intersecting $U_{i}$ twice or an annulus disjoint from $U_{i}$. Suppose $D$ is a $P$-compressing disk for $F_{j}$ as in the definition, and let $\alpha=D \cap P$. Since $\beta=\left|D \cap F_{j}\right|$ is essential on $F_{j}-U_{i}$, the two points $\partial \alpha=\partial \beta$ lie on distinct components of $F_{j} \cap P$, and hence an isotopy of $F$ via $D$ would create a surface $F^{\prime}$ that has the same or smaller complexity than $F$, and yet one of the faces $F_{j}^{\prime}$ deformed from $F_{j}$ has $i\left(F_{j}^{\prime}, U_{i}\right)>0$, which is a contradiction of (ii).

Lemma 4.3. If $F$ is a special surface in $E(K)$ then it is not a punctured sphere.

Proof. This follows from the proof of Lemma 4.2(ii). If $\hat{F}$ is a sphere, then $2=$ $\chi(\hat{F})=\sum i\left(F_{j}, U_{i}\right)$, which is a contradiction since $i\left(F_{j}, U_{i}\right) \leq 0$ for all $i, j$.

Lemma 4.4. Let $F$ be an essential punctured torus in $E(K)$ with integer boundary slope. Then $F, P_{1}, P_{2}$ can be isotoped so that $F$ is a special surface.

Proof. We may assume that $(|F \cap \partial P|,|F \cap P|)$ is minimal up to isotopy, so Lemma 4.2 applies, and we have $i\left(F_{k}, U_{i}\right)=0$ for any component $F_{k}$ of $F \cap E\left(T_{i}\right)$. We show below that $P_{i}$ can be isotoped so that each component $D$ of $F \cap E\left(T_{i j}\right)$ is a tight disk. In that case, by Lemma 3.3, we will have $i\left(D, U_{i}\right) \leq 0$; since $F_{k}$ is a union of such disks, by the Additivity Lemma 3.2 we will have $i\left(D, U_{i}\right)=0$ for all $D$, and hence by Lemma $3.3 D$ is a special disk in $E\left(T_{i j}\right)$, which will complete the proof.

Let $Q_{i}=F \cap E\left(T_{i}\right)$. Isotope $P_{i}$ so that $\left(\left|Q_{i} \cap \partial P_{i}\right|,\left|Q_{i} \cap P_{i}\right|\right)$ is minimal. This implies that for $R=P_{-}\left(T_{i 1}\right), P_{+}\left(T_{i 2}\right)$ or a component of $U\left(T_{i j}\right)$, each arc 
component of $Q_{i} \cap R$ is essential on $R$. By Lemma 4.2, $Q_{i}$ is incompressible and $P$-incompressible, so the above minimality and the $\partial$-incompressibility of $F$ imply that each component of $Q_{i} \cap P_{i}$ is also essential on $P_{i}=P_{+}\left(T_{i 1}\right)=P_{-}\left(T_{i 2}\right)$. Therefore all components of $F \cap E\left(T_{i j}\right)$ are tight. It remains to show that each component of $F \cap E\left(T_{i j}\right)$ is a disk.

By Lemma 2.2, $P_{i}$ is incompressible and $\partial$-incompressible in $E\left(T_{i}\right)-U_{i}$, so no component of $P_{i} \cap Q_{i}$ is a trivial loop on $Q_{i}$, or an arc that is trivial on $Q_{i}-U_{i}$ in the sense that it cuts off a disk on $Q_{i}$ disjoint from $U_{i}$. Thus if a component $F_{k}$ of $Q_{i}$ is a disk, then $P_{i}$ cuts $F_{k}$ into disks. Similarly, if $F_{k}$ is an annulus, then no component of $F_{k} \cap P_{i}$ is an inessential arc or inessential circle on $F_{k}$. It now suffices to show that $\partial F_{k} \cap P_{i} \neq \varnothing$, because then $F_{k} \cap P_{i}$ is a nonempty set of essential arcs on $F_{k}$, cutting it into disks.

Let $F_{k}$ be an annulus component of $Q_{i}$, with $\partial F_{k}$ disjoint from $P_{i}$. This implies that $F_{k} \cap U_{-}(T)=\varnothing$, because each component of $F_{k} \cap U_{-}\left(T_{i}\right)$ is a component of $F \cap U_{-}\left(T_{i}\right)$, which intersects $P_{i}$ at two points. Since $i\left(F_{k}, U_{i}\right)=0$, we also have $F_{k} \cap U_{+}\left(T_{i}\right)=\varnothing$. Therefore $\partial F_{k} \subset P$. No component $C^{\prime}$ of $\partial F_{k}$ is parallel to a component $C$ of $\partial P$, since otherwise the arc components of $F \cap P$ with endpoints on $C$ would lie in the annulus between $C$ and $C^{\prime}$, and hence would be trivial arcs, and there are $|\partial F| / 2>0$ of them, which can be used to $\partial$-compress $F$, a contradiction to the fact that $F$ is essential. Therefore the two components of $\partial F_{k}$ are both parallel to the circle $P_{i} \cap P$, and hence bound an annulus $A$ on $P$. By Lemma 4.2(iii), $F_{k}$ is incompressible, and by [Wu 1996a, Lemma 3.3] $E\left(T_{i}\right)$ is a handlebody, so $F_{k} \cup A$ must bound a solid torus $V$ in $E\left(T_{i}\right)$. Now $A$ cannot be meridional on $V$ because $P$ is incompressible, and $A$ cannot run more than once along the longitude of $V$, since otherwise the union of $V$ and a regular neighborhood of a disk on $\partial B_{i}$ bounded by a component of $\partial F_{k}$ would be a punctured lens space in the 3-ball $B_{i}$, which is absurd. It follows that $A$ is longitudinal on $\partial V$ and hence $F_{k}$ is $P$-compressible, which contradicts Lemma 4.2 (iii).

Proposition 4.5. Suppose $K$ is an arborescent knot of type $I I$, and suppose $E(K)$ contains an essential punctured torus with integer boundary slope. Then $K$ is the union of $T_{1}, T_{2}$, each of which is weakly equivalent to $T\left(\frac{1}{3}, \frac{1}{2}\right)$ or $T\left(-\frac{1}{3}, \frac{1}{2}\right)$.

Proof. By definition, $K$ is the union of $T_{1}, T_{2}$ with $T_{i}=T\left(p_{i} / q_{i}, \frac{1}{2}\right)=T_{i 1}+T_{i 2}$. By Lemma 4.4, we may assume that $F$ intersects each $E\left(T_{i j}\right)$ in special disks. If some $q_{i}>3$, then the only special disk in $E\left(T_{i 1}\right)$ is of type (1), and hence each component of $F \cap P_{i}$ is an arc with only one endpoint on the circle $P_{i} \cap P$, where $P_{i}$ is the twice-punctured disk $E\left(T_{i 1}\right) \cap E\left(T_{i 2}\right)$. From the definition, we see that the only special disks in $E\left(T_{i 2}\right)=E\left(T\left(\frac{1}{2}\right)\right)$ intersecting $P_{i}$ in such arcs are those of type (4), which are disjoint from $U_{+}\left(T_{2}\right)$. Therefore $F \cap U_{+}\left(T_{i}\right)=\varnothing$, which is a contradiction, since $F$ must intersect each $U_{ \pm}\left(T_{i}\right)$ in exactly $|\partial F|>0$ arcs. 


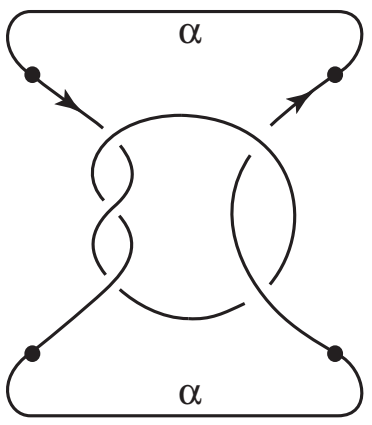

(1)

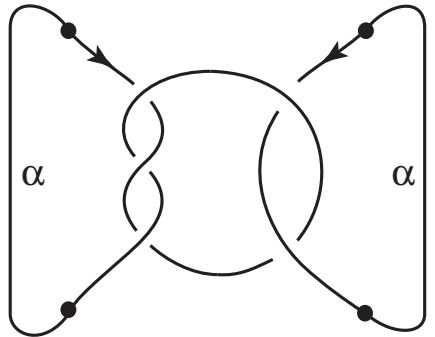

(2)

Figure 3

\section{Boundary slopes of special surfaces}

Let $T=\left(B^{3}, \tau\right)$ be a tangle, with $B^{3}$ identified to $\hat{R}_{-}^{3}$. We assume that $\tau$ is oriented. Let $\alpha$ be a pair of arcs on $R^{2}-\operatorname{Int} I^{2}$, shown in Figure 3(1) when the orientations of $\tau$ are opposite near the two upper endpoints, or in Figure 3(2) otherwise. Then $\hat{\tau}=\tau \cup \alpha$ is a link in $R^{3}$ with orientation induced from that of $\tau$.

Let $\gamma$ be a set of essential arcs on $U(T)$, and assume that $n=\left|\gamma \cap U_{-}(T)\right|=$ $\left|\gamma \cap U_{+}(T)\right|$. Each component of $\gamma$ is isotopic in $N(\tau)$ to a component of $\tau$, so the orientation of $\tau$ induces an orientation on $\gamma$. Let $p: R_{-}^{3} \rightarrow R^{2}=\partial R^{3}$ be the standard projection. We always assume that $\tau$ and $\gamma$ are in regular position in the sense that

- $p(\tau) \subset I^{2}$, where $I=[-1,1]$;

- $p: \tau \cup \gamma \rightarrow R^{2}$ is an immersion with only double crossings; and

- $p(\gamma) \cap \alpha=\varnothing$.

The third condition is to guarantee that all crossings between $p(\gamma)$ and $p(\tau \cup \alpha)$ appear inside of the square $I^{2}$, so if we close up $\gamma$ by arcs parallel to $\alpha$ to obtain $\hat{\gamma}$, then the linking number between $\hat{\gamma}$ and $\hat{\tau}=\tau \cup \alpha$ can be calculated using crossings between $\tau$ and $\gamma$.

Since $\tau$ and $\gamma$ are oriented, each crossing is assigned a sign according to the right hand rule, as given in [Rolfsen 1990, p. 132].

Definition 5.1. (i) The relative linking number between $\tau$ and $\gamma$, denoted by $l k(\tau, \gamma)$, is the sum of the signs of crossings at which $\gamma$ passes below $\tau$.

(ii) Let $F$ be a surface in $E(T)$ such that $F \cap U(T)$ is in regular position. Then the relative framing of $F$ in $E(T)$ is defined as $\theta(F)=l k(\tau, F \cap U(T))$. 
An isotopy of $\tau \cup \gamma$ is a regular isotopy if $\tau \cup \gamma$ is in regular position at any time during the isotopy. Similarly, an isotopy of a surface $F$ in $E(T)$ is a regular isotopy if its restriction to $F \cap U(T)$ is a regular isotopy.

Consider the four disks $\cup D_{i}=R^{2}-\operatorname{Int} P(T)$. Since $\gamma$ has $n$ endpoints on each $\partial D_{i}$, we can connect $\partial \gamma$ by $2 n \operatorname{arcs} \gamma^{\prime}$ on $\partial N(\hat{\tau})$ lying in the upper half-space $R_{+}^{3}$. Define $\hat{\gamma}=\gamma \cup \gamma^{\prime}$, with orientation induced by that of $\gamma$. Since $\hat{\tau} \cup \hat{\gamma}$ is an oriented link, the linking number $l k(\hat{\tau}, \hat{\gamma})$ is well defined.

Lemma 5.2. (i) $l k(\tau, \gamma)=l k(\hat{\tau}, \hat{\gamma})$.

(ii) $l k(\tau, \gamma)$ and $\theta(F)$ are regular isotopy invariants .

(iii) Let $\psi$ be a rotation of $R_{-}^{3}$ along the $z$-axis by an angle of $\pi / 2$, deforming $\tau$ to $\tau^{\prime}$ and a surface $F$ to $F^{\prime}$. Then $\theta(F)=\theta\left(F^{\prime}\right)$.

(iv) Suppose $F_{j}$ are the components of $F$ in $E(T)$. Then $\theta(F)=\sum \theta\left(F_{j}\right)$.

Proof. (i) It is well known that $l k(\hat{\tau}, \hat{\gamma})$ can be calculated as the sum of the signs of crossings at which $\hat{\gamma}$ passes below $\hat{\tau}$. By definition, $\gamma$ does not pass below $\alpha$, and $\gamma^{\prime}$ does not pass below $\hat{\gamma}$ because it lies in $R_{+}^{3}$ while $\hat{\gamma}$ lies in $R_{-}^{3}$. Therefore the crossings at which $\hat{\gamma}$ passes below $\hat{\tau}$ are exactly where $\gamma$ passes below $\tau$, and the result follows.

(ii) A regular isotopy does not change the relative position of $\partial \tau$ to $\alpha$, and hence it extends to an isotopy of $\hat{\tau} \cup \hat{\gamma}$, and the result follows from (i) because the linking number of a link is an isotopy invariant.

(iii) This follows from the definition, because $\psi$ gives a sign preserving one-to-one correspondence between the crossings.

(iv) This follows from the definition.

Let $T=T_{1}+T_{2}$, where $T_{1}=T\left(\frac{1}{3}\right)$ and $T_{2}=T\left(-\frac{1}{2}\right)$. Let $F$ be a special surface in $E(T)$ intersecting each $U_{ \pm}(T)$ in $n$ arcs. By definition, each component of $F \cap E\left(T_{i}\right)$ is a special disk. We may assume that $F$ is a union of $a_{i}$ copies of $A_{i}$ for $i=1, \ldots, 6$. From Figure 1 we see that $F \cap P\left(T_{i}\right)$ is as shown in Figure 4(i), $i=1,2$, where an arc with label $a_{j}$ indicates $a_{j}$ parallel copies of that arc.

Lemma 5.3. Let $r=n / 2$, and $s=a_{6}$. Then there is a special surface $F$ in $E(T)$ consisting of $a_{k}$ copies of disks of type $(k)$ and intersecting each $U_{i}(T)$ at $n$ arcs, if and only if the nonnegative integers $a_{k}$ satisfy the following equations.

(i) $n=2 r$ is even.

(ii) $a_{1}=r-s$.

(iii) $a_{2}=a_{3}=r+s$.

(iv) $a_{4}=a_{5}=a_{1}+a_{2}=a_{1}+a_{3}=n$.

(v) $a_{6}=s$. 
Proof. Suppose $F$ is such a surface. Then (v) is from definition, and (iv) follows from the fact that $F$ intersects each boundary component of $P$ at $n$ points. By (iv) we have $a_{2}=a_{3}$. Let $P^{\prime}=P_{+}\left(T_{1}\right)=P_{-}\left(T_{2}\right)$ be the twice-punctured disk cutting $E(T)$ into $E\left(T_{1}\right)$ and $E\left(T_{2}\right)$. Then on $\partial E\left(T_{1}\right)$, there are $a_{2}+a_{3}$ arcs of $F \cap P^{\prime}$ with both endpoints on the circle $P^{\prime} \cap P(T)$, while there are $a_{5}+2 a_{6}$ such arcs on $\partial E\left(T_{2}\right)$. Hence $2 a_{2}=a_{2}+a_{3}=a_{5}+2 a_{6}=n+2 s$, which shows that $n=2 r$ is even, and $a_{2}=a_{3}=r+s$. Item (ii) follows from this and the equation $a_{1}+a_{2}=n=2 r$, as shown in Figure 4(a).

Conversely, given a set of nonnegative numbers $a_{k}$ satisfying the above equations, let $E_{k}$ be $a_{k}$ copies of disks of type $(k)$. Then one can check that the arcs $\left(E_{1} \cup E_{2} \cup E_{3}\right) \cap P^{\prime}$ are isotopic to the $\operatorname{arcs}\left(E_{4} \cup E_{5} \cup E_{6}\right) \cap P^{\prime}$ on $P^{\prime}$, and hence one can glue these together to form a surface $F$ in $E(T)$.

The curves in parts (a) and (b) of Figure 4 can be represented by the weighted train tracks shown in parts (c) and (d), where an arc of the train track with weight $x$ represents $x$ parallel copies of that arc. The weights on the train tracks are calculated using the above lemma.

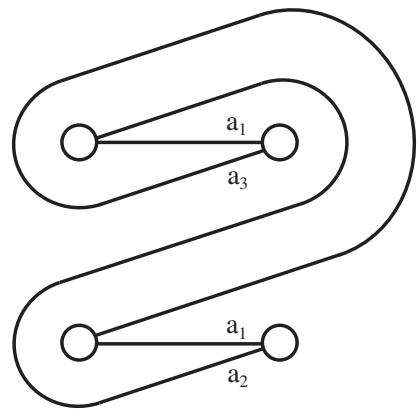

(a)

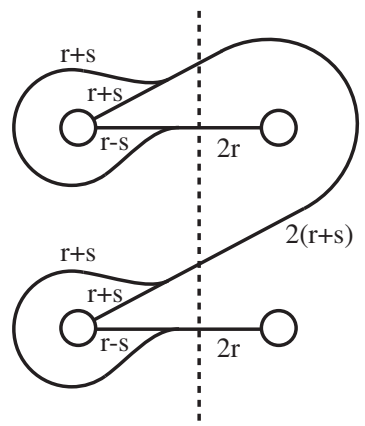

(c)

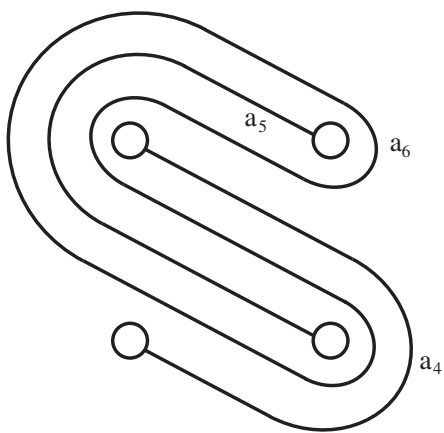

(b)

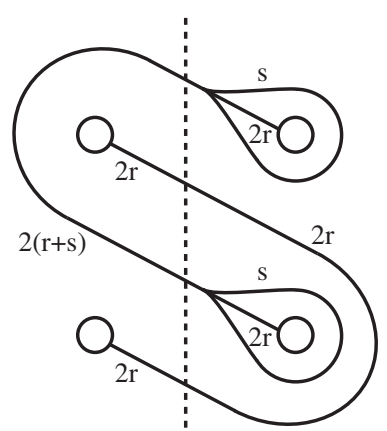

(d)

Figure 4 


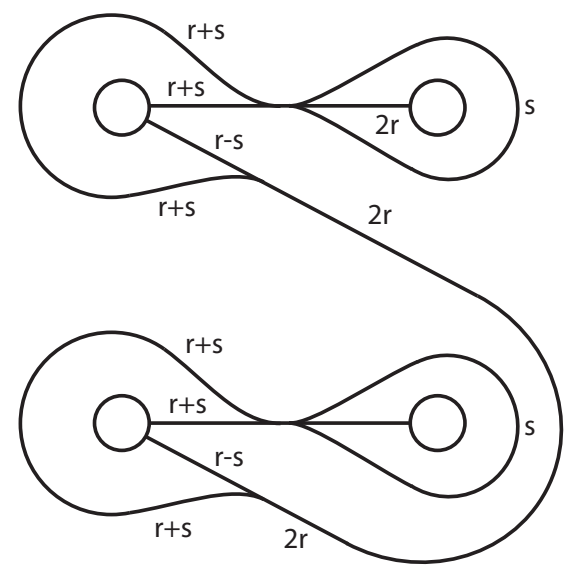

(a)

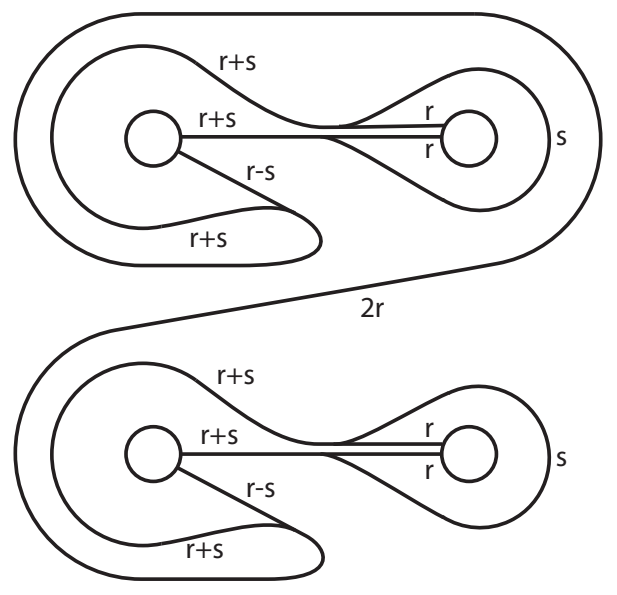

(b)

Figure 5

Recall that $T\left(\frac{1}{3},-\frac{1}{2}\right)=T_{1}+T_{2}$ is formed by gluing $P_{+}\left(T_{1}\right)$ to $P_{-}\left(T_{2}\right)$ using a reflection map along the $y$-axis on $R^{2}$. Since the train tracks on these two halfplanes match each other under this reflection, after gluing we obtain a special surface $F$ in $E(T)$ with $F \cap P$ represented by the train track $\gamma$ in Figure 5(a).

To simplify the diagram, we perform a counterclockwise full twist on both the top two tangle endpoints and the bottom two tangle endpoints. This is equivalent to twisting the two lower endpoints by four left-hand half-twists, so by definition it deforms the tangle $T\left(\frac{1}{3},-\frac{1}{2}\right)$ to the tangle $T\left(\frac{1}{3},-\frac{1}{2} ; 4\right)$ defined in Section 2 . The train track, after splitting along two edges, becomes that in Figure 5(b).

After a further splitting and isotopy, we obtain the train track $\gamma$ in Figure 6(a). Up to isotopy we can move the end points of $\gamma$ around $\partial P$, but so far we have not done that. By moving some endpoints of train track around $\partial P$, we obtain the one in Figure 6(b).

The two strings of $T=T( \pm 1 / q)$ are said to be consistently oriented if they both run from the upper endpoints to the lower endpoints or both from the lower endpoints to the upper endpoints. For $T\left(\frac{1}{3}\right)$ we will always assume that its two strings are consistently oriented. For $T\left(-\frac{1}{2}\right)$, we introduce a new variable $\epsilon$ and set $\epsilon=1$ if the two strings of $T\left(-\frac{1}{2}\right)$ are oriented consistently, and $\epsilon=-1$ otherwise. Recall that a surface $F$ in $E(T)$ is regular if $F \cap U(T)$ is a set of regular curves on $U(T)$.

Lemma 5.4. Let $A_{k}$ be a regular special disk such that $A_{k} \cap P(T)$ is the curve in part $(k)$ of Figure 1.

(i) $\theta\left(A_{1}\right)=6, \theta\left(A_{2} \cup A_{3}\right)=4, \theta\left(A_{4} \cup A_{5}\right)=-2 \epsilon$, and $\theta\left(A_{6}\right)=0$. 


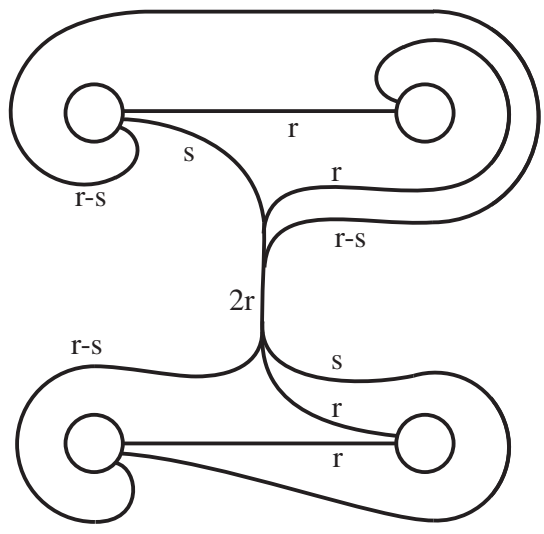

(a)

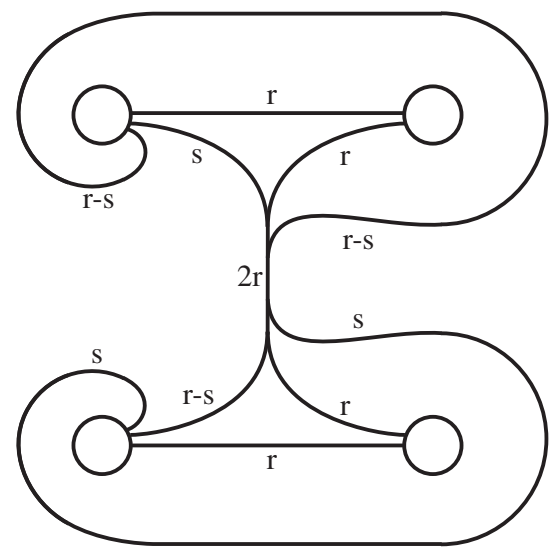

(b)

\section{Figure 6}

(ii) A special surface $F$ in $T\left(\frac{1}{3},-\frac{1}{2}\right)$ is isotopic to a regular surface $F^{\prime}$ such that $\partial F^{\prime} \cap P$ is represented by the weighted train track in Figure $5(a)$, and $\theta\left(F^{\prime}\right)=$ $(5-2 \epsilon) n-2 s$, where $s=a_{6}$ is the number of type (6) special disks in $F$.

(iii) A special surface $F$ in $T\left(\frac{1}{3},-\frac{1}{2} ; 4\right)$ is isotopic to a regular surface $F^{\prime}$ such that $F^{\prime} \cap P$ is carried by the weighted train track in Figure $6(b)$, and $\theta(F)=$ $(6-4 \epsilon) n-2 s$.

Proof. (i) follows by drawing the curves $\gamma$ of $\partial A_{i}$ on $U(T)$ and counting the signed crossings where $\gamma$ passes below $\tau$. We omit the details.

(ii) By definition, $F$ is the union of $a_{k}$ copies of $A_{k}$, which can be put in regular position. We leave it to the reader to check that the regular surfaces in $E\left(T\left(\frac{1}{3}\right)\right)$ and $E\left(T\left(-\frac{1}{2}\right)\right)$ can be combined together to create the surface $F$ in $E\left(T\left(\frac{1}{3},-\frac{1}{2}\right)\right)$ without creating new crossings between $F \cap U(T)$ and $\tau$. Since $a_{2}=a_{3}$ and $a_{4}=a_{5}$, we have $\theta(F)=a_{1} \theta\left(A_{1}\right)+a_{2} \theta\left(A_{2} \cup A_{3}\right)+a_{4} \theta\left(A_{4} \cup A_{5}\right)+a_{6} \theta\left(A_{6}\right)$. It follows from (i) and Lemma 5.3 that

$$
\theta(F)=6 a_{1}+4 a_{2}-2 \epsilon a_{4}=6(r-s)+4(r+s)-2 \epsilon n=(5-2 \epsilon) n-2 s .
$$

(iii) By definition, $T=T\left(\frac{1}{3},-\frac{1}{2}\right.$; 4) is obtained from $T\left(\frac{1}{3},-\frac{1}{2}\right)$ by two counterclockwise full twists of the two lower endpoints of the tangle, which deforms the surface $F^{\prime \prime}$ in (ii) to a new surface $F^{\prime}$ in $E(T)$ with boundary curve represented by the train track shown in Figure 6(a). After the twists, each arc component of $\alpha^{\prime}=F^{\prime} \cap \partial N(\tau)$ passes below $\tau$ four more times than $\alpha^{\prime \prime}=F^{\prime \prime} \cap \partial N(\tau)$ does, two of those times in the positive direction, and the other two in the positive direction if and only if $\epsilon=-1$. (The two strings twisted have the same orientation if and 
only if the two strings in the tangle $T\left(-\frac{1}{2}\right)$ have opposite orientation.) Hence $\theta\left(F^{\prime}\right)=\theta\left(F^{\prime \prime}\right)+(2-2 \epsilon) n$, so by (ii) we have $\theta\left(F^{\prime}\right)=(7-4 \epsilon) n-2 s$.

We now perform an isotopy of $F^{\prime}$ to obtain the surface $F$ whose boundary curve on $P$ is carried by the train track in Figure 6(b). To do this one needs to turn $2 r=n$ endpoints of $F \cap P$ on $\partial P$ clockwise for an angle of almost $2 \pi$. The isotopy on each endpoint creates one more crossing at which $\alpha^{\prime}$ passes below $\tau$, and it in the negative direction. Therefore we have $\theta(F)=\theta\left(F^{\prime}\right)-n=(6-4 \epsilon) n-2 s$.

Lemma 5.5 can be used to calculate the boundary slope of a surface in $E(K)$.

Lemma 5.5. Suppose $F_{i}$ is a regular surface in $E\left(T_{i}\right)$. Let $\eta: \partial B_{1}=\hat{R}^{2} \rightarrow \hat{R}^{2}=$ $\partial B_{2}$ be the reflection along the $y$-axis, such that $\eta\left(F_{1} \cap P\left(T_{1}\right)\right)=F_{2} \cap P\left(T_{2}\right)$. Let $\left(S^{3}, K\right)=\left(B_{1}, \tau_{1}\right) \cup_{\eta}\left(B_{2}, \tau_{2}\right)$ with orientation of $\tau_{i}$ induced by that of $K$, and let $F=F_{1} \cup_{\eta} F_{2}$. Suppose $F$ has $m$ boundary components with slope $p / q$, where $p, q$ are coprime and $q>0$. Then $m p=\theta\left(F_{1}\right)+\theta\left(F_{2}\right)$. In particular, $q=1$ if and only if $\theta\left(F_{1}\right)+\theta\left(F_{2}\right) \equiv 0 \bmod n$, where $n=m q$ is the number of times $\partial F$ intersects each meridian of $K$.

Proof. We can shift $T_{1}=\left(B^{3}, \tau_{1}\right)$ to the left and $T_{2}=\left(B^{3}, \tau_{2}\right)$ to the right so that $\tau_{1}, \tau_{2}$ are separated by the $y z$-half-plane in $B^{3}=R_{-}^{3}$. Now $K \subset R^{3}$ is isotopic to the knot $K^{\prime}$ obtained by adding four arcs on $R^{2}$, each connecting an endpoint $p_{i}$ of $\tau_{1}$ to $\eta\left(p_{i}\right)$ on $\tau_{2}$, with two below the line $y=-1$ and the other two above the line $y=1$. We may also assume that near $\partial \tau_{i}$ these $\operatorname{arcs}$ match the $\operatorname{arcs} \alpha$ in Figure 3, so they are disjoint from the projection of $F_{i} \cap U\left(T_{i}\right)$ because $F_{i}$ are regular. The isotopy from $K$ to $K^{\prime}$ extends to an isotopy which deforms $F$ in $E(K)$ to the surface $F^{\prime}$ in $E\left(K^{\prime}\right)$ obtained from $F_{1} \cup F_{2}$ by connecting their boundary on $R^{2}$ by bands in the upper half-space. More explicitly, let $C=F_{1} \cap R^{2}$ and embed $C \times I$ in $R_{+}^{3} \cap E\left(K^{\prime}\right)$ so that $C \times\{-1\}=F_{1} \cap R^{2}, C \times\{1\}=F_{2} \cap R^{2}$, and $\partial C \times I$ lies on $\partial N\left(K^{\prime}\right)$. Then $F^{\prime}=F_{1} \cup(C \times I) \cup F_{2}$. Note that $(\partial C) \times I$ does not pass below $K^{\prime}$, so $l k\left(K^{\prime}, \partial F^{\prime}\right)$ is the sum of the signs of crossings where $\partial F_{i}$ passes below $\tau_{i}$, and hence $m p=l k(K, \partial F)=l k\left(K^{\prime}, \partial F^{\prime}\right)=\theta\left(F_{1}\right)+\theta\left(F_{2}\right)$.

If $q=1$ then $m p \equiv 0 \bmod n=m q$. Conversely, if $m p \equiv 0 \bmod n=m q$ then $p$ is a multiple of $q$. Since $p, q$ are coprime and $q>0$, this is possible only if $q=1$.

We now assume that $K$ is a type II knot and $F$ is an essential punctured torus in $E(K)$ with integer boundary slope. By Proposition $4.5, K$ is the union of two tangles $T_{1}, T_{2}$, each weakly equivalent to $T\left(\frac{1}{3},-\frac{1}{2}\right)$ or $T\left(-\frac{1}{3}, \frac{1}{2}\right)$. Hence, up to weak isotopy, we may assume that $T_{i}=T\left(\frac{1}{3},-\frac{1}{2} ; 4\right)$ or its mirror image $T\left(-\frac{1}{3}, \frac{1}{2} ;-4\right)$. By Lemma 4.4, we may assume that $F_{i}=F \cap E\left(T_{i}\right)$ is a special surface, so by Lemma 5.4(iii) we may assume that $F_{i}$ is regular, and $F_{i} \cap P\left(T_{i}\right)$ is represented by the train track $\gamma_{i}$ in Figure 6(b) if $T_{i}=T\left(\frac{1}{3},-\frac{1}{2} ; 4\right)$, and by its reflection along the $y$-axis if $T_{i}=T\left(-\frac{1}{3}, \frac{1}{2} ;-4\right)$. The gluing map $\eta: \partial B_{2} \rightarrow \partial B_{1}$ could be any orientation-reversing map that maps $P\left(T_{2}\right)$ to $P\left(T_{1}\right)$ and $F_{1} \cap P\left(T_{1}\right)$ to $F_{2} \cap P\left(T_{2}\right)$. 


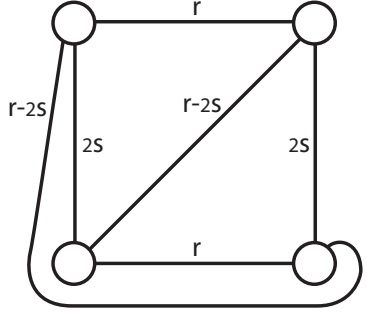

(1)

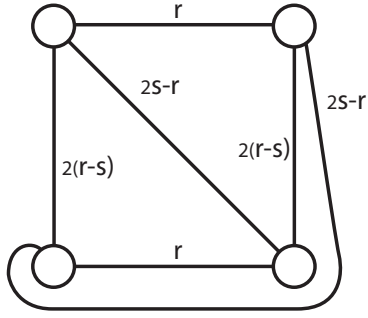

(2)

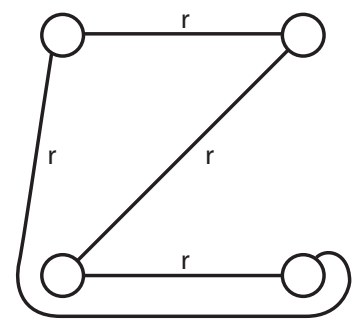

(3)

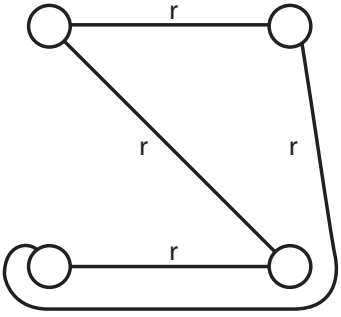

(4)

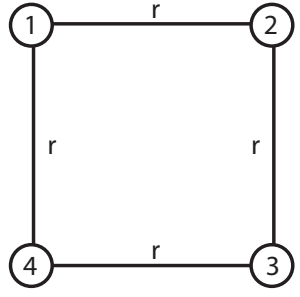

(5)

Figure 7

As in Lemma 5.3, let $n$ be the number of times $F$ intersects a meridian of $K$ on $N(K), r=n / 2$, and let $s_{i}$ be the number of type (6) special disks in $F_{i}$. There are five possible ways to split $\gamma_{i}$, according to the values of $s_{i}$ :
(1) $r-s_{i}>s_{i}>0$
(2) $s_{i}>r-s_{i}>0$
(3) $s_{i}=0$
(4) $r-s_{i}=0$
(5) $r-s_{i}=s_{i}$

One can check that for $T_{i}=T\left(\frac{1}{3},-\frac{1}{2} ; 4\right)$ the train track in Figure 6(b) splits to $\gamma_{i}$ in the corresponding parts of Figure 7, where $s=s_{i}$. When $T_{i}=T\left(-\frac{1}{3}, \frac{1}{2} ;-4\right)$, $\gamma_{i}$ is the reflection of those in the figure along the $y$-axis. We say that $\gamma_{i}$ is of type $(k)$ if it is the one shown in part $(k)$ of Figure 7.

Lemma 5.6. For each $i, s_{i} \in\{0, r, r / 2\}$. Hence $\gamma_{i}$ is not of type (1) or (2).

Proof. We need to show that $\gamma_{i}$ cannot be of type (1) or (2). Because of symmetry we may assume without loss of generality that $T_{1}=T\left(\frac{1}{3},-\frac{1}{2} ; 4\right)$, and $\gamma_{1}$ is of type (1). By Lemma 5.4(iii) we have $\theta\left(F_{1}\right)=(6-4 \epsilon) n-2 s_{1}$.

The graph in part (1) is not homeomorphic to those in (3)-(5), so $\gamma_{2}$ must be of type (1) or (2). Let $\eta: \partial B_{2} \rightarrow \partial B_{1}$ be the gluing map, which is orientationreversing. Since the two horizontal edges of $\gamma_{i}$ have higher weights than the other edges, $\eta$ must map horizontal edges to horizontal edges. Without loss of generality we may assume that $\eta$ maps the upper horizontal line of $\gamma_{2}$ to the upper horizontal line of $\gamma_{1}$ by a reflection along the vertical axis. This fully determines $\eta$ on $\gamma_{2}$. 
First assume that $T_{2}=T\left(\frac{1}{3},-\frac{1}{2} ; 4\right)$. If $\gamma_{2}$ is the one in Figure $7(2)$, then $\eta$ is simply a reflection along the vertical line, and hence it maps the two right vertices of $\gamma_{2}$ to the two left vertices of $\gamma_{1}$, but since the two left (right) vertices of $\gamma_{i}$ belong to the same component of $\tau_{i}, K$ would be a link of two components, which is a contradiction. Therefore $\gamma_{2}$ must be the graph in Figure $7(1)$, which is redrawn in Figure 8(1). One can modify the graph by turning the lower horizontal edge clockwise by a half-twist to obtain the one in Figure 8(2), then isotope some of the edge endpoints at the two lower boundary components of $P$ around to obtain the graph in Figure 8(3). The map $\eta$ is the composition of this isotopy followed by a reflection along a vertical line.

The isotopies above are not regular isotopies. They have changed the relative framing of the surface $F_{2}$. By Lemma 5.4(iii), the framing of $F_{2}$ with boundary graph in Figure 8(1) is given by $(6-4 \epsilon) n-2 s_{2}$. After twisting the two lower vertices of the graph by a half-twist, each boundary arc of $F_{2}$ on the tubes $\partial E\left(T_{2}\right)-P$ passes below the part of $\tau$ near the vertex 3 in the figure once in the negative direction, but does not pass below the other string of $\tau$, and hence the new framing is $(4-4 \epsilon) n-2 s_{2}$. The isotopy from Figure 8(2) to 8(3) moves $r$ edge endpoints clockwise and another $r$ edge endpoints counterclockwise around vertex 3 and 4 respectively, and hence will not change the relative framing. Therefore we have $\theta\left(F_{2}\right)=(4-4 \epsilon) n-2 s_{2}$ for the surface $F_{2}$ corresponding to Figure 8(3).

In order to glue $F_{2}$ to $F_{1}$ by $\eta$, the weight of the left vertical edge in Figure 8(3) must match the weight of the right vertical edge of Figure 7(1), and hence we have $r-2 s_{2}=2 s_{1}$. Thus $\theta\left(F_{1}\right)=(6-4 \epsilon) n-\left(r-2 s_{2}\right)$, so

$$
\begin{aligned}
\theta\left(F_{1}\right)+\theta\left(F_{2}\right) & =\left((6-4 \epsilon) n-\left(r-2 s_{2}\right)\right)+\left((4-4 \epsilon) n-2 s_{2}\right) \\
& =(10-8 \epsilon) n-r \equiv r \quad \bmod n .
\end{aligned}
$$

Since $r=n / 2$, this is a contradiction to Lemma 5.5 and the assumption that $F$ has integer boundary slope.

Lemma 5.7. $s_{1}=s_{2}=r / 2$, so both $\gamma_{i}$ are of type (5).

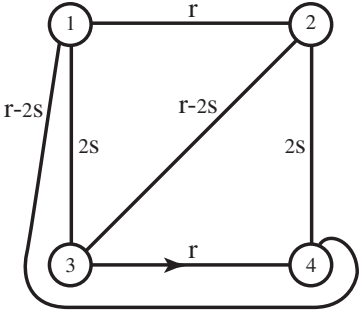

(1)

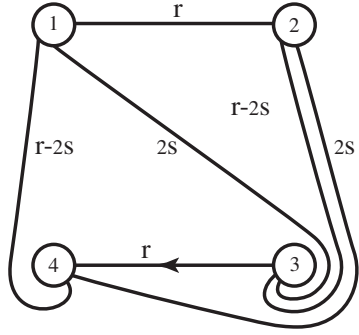

(2)

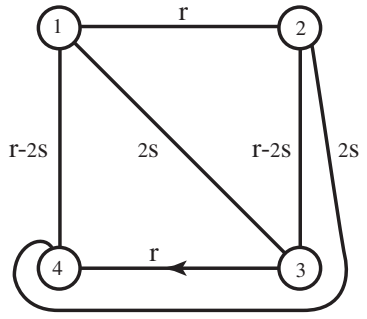

(3)

Figure 8 
Proof. If $\gamma_{i}$ is of type (3) or (4), then the endpoints of a string of $\tau_{i}$ are separated by those of the other string on $\gamma_{i}$, which is a circle. Hence if both $\gamma_{i}$ are of type (3) or (4) then $K=\tau_{1} \cup \tau_{2}$ would be a link of two components, which is a contradiction. Therefore at least one $\gamma_{i}$, say $\gamma_{1}$, is of type (5). If the result is not true then $\gamma_{2}$ must be of type (3) or (4). We assume it is of type (3). The other case is similar.

By the same proof as in that of Lemma 5.6, we can isotope $\gamma_{2}$ by twisting the lower level edge of $\gamma_{2}$ by a half-twist, followed by an isotopy that moves some endpoints of $\gamma_{2}$ around $\partial P$, to change $\gamma_{2}$ to a graph of type (5). As in the proof of Lemma 5.6, this will not change $\theta\left(F_{2}\right) \bmod n$. By Lemma 5.4(iii), we have $\theta\left(F_{1}\right)+\theta\left(F_{2}\right) \equiv-2 s_{1}-2 s_{2} \bmod n$. Since $\gamma_{2}$ is of type (3), we have $s_{2}=0$, and since $\gamma_{1}$ is of type (5), we have $2 s_{1}=r=n / 2$. Hence $\theta\left(F_{1}\right)+\theta\left(F_{2}\right) \equiv r \bmod n$, which by Lemma 5.5 implies that the boundary slope of the punctured torus $F$ is not an integer slope, a contradiction.

Proposition 5.8. Let $K$ be a type II knot, and let $F$ be an essential punctured torus in $E(K)$ with integer boundary slope $\delta$. Then $|\partial F|$ is a multiple of 4 , and $(K, \delta)=$ $\left(K_{1}, 3\right),\left(K_{2}, 0\right)$ or $\left(K_{3},-3\right)$, where $K_{i}$ are the knots defined in Definition 2.3.

Proof. By Proposition 4.5, we have $\left(S^{3}, K\right)=T_{1} \cup T_{2}$, where each $T_{1}$ is weakly equivalent to $T\left(\frac{1}{3}, \frac{1}{2}\right)$ or $T\left(-\frac{1}{3}, \frac{1}{2}\right)$. Up to weak equivalence we may assume that each $T_{i}$ is either $T\left(\frac{1}{3},-\frac{1}{2} ; 4\right)$ or $T\left(-\frac{1}{3}, \frac{1}{2} ;-4\right)$.

Denote by $u_{1}, \ldots, u_{4}$ the four disks $\partial B_{i}-\operatorname{Int} P\left(T_{i}\right)$, which we consider as fat vertices. By Lemma 5.7, the train track $\gamma_{1}$ is of type (5), so it is a cycle containing those four vertices, labeled in cyclic order, as shown in Figure 7(5). Similarly, $\gamma_{2}$ contains the vertices $v_{1}, \ldots, v_{4}$ in the same order. Orient $\gamma_{i}$ clockwise. Let $\eta: \partial B_{1} \rightarrow \partial B_{2}$ be the gluing map. Then up to isotopy $\eta$ is determined by its restriction on $\gamma_{1}$, which is then determined by the image of $u_{1}$ and whether $\left.\eta\right|_{\gamma_{1}}$ is orientation-preserving or not. Although $\eta$ must be orientation-reversing on $\partial B_{1}$, it may map the disk inside of $\gamma_{1}$ to the disk outside of $\gamma_{2}$, so $\left.\eta\right|_{\gamma_{1}}$ could be orientationpreserving.

Since $K$ is a knot, the endpoints of a string of $\tau_{2}$ must be mapped to endpoints of different strings of $\tau_{2}$, which excludes four possible $\eta$. Also, if $\tau_{i}$ is considered as lying in a pillowcase $B_{i}$, then from Figure 2(a) one can see that a $\pi$-rotation along a horizontal axis will preserve the tangle. One can now easily check that all the four possible choices of $\eta$ give rise to the same knot, so we may assume that $\eta$ is obtained by rotating $\gamma_{2}$ counterclockwise by an angle of $\pi / 2$ and then reflecting along a vertical line, as described in Definition 2.3. Therefore $K$ is one of the three knots in the statement.

The surface $F$ is cut into $F_{i}$ in $E\left(T_{i}\right)$. By Lemma 5.2(iii), the $(\pi / 2)$-rotation above will not change $\theta\left(F_{1}\right)$, so by Lemma 5.5 the boundary slope of $F$ is given by $\left(\theta\left(F_{1}\right)+\theta\left(F_{2}\right)\right) / n$. Examining the orientation of the strings in $\tau_{i}$ we see that 
they are consistently oriented in the tangle $T\left( \pm \frac{1}{2}\right)$, and hence $\epsilon=1$ for both $T_{i}$. Since $\gamma_{i}$ are of type (5), by definition we have $2 s_{i}=r$, so by Lemma 5.4(iii) we have $\theta\left(F_{i}\right)=(6-4 \epsilon) n-2 s_{i}=2 n-r$ if $T_{i}=T\left(\frac{1}{3},-\frac{1}{2} ; 4\right)$, and $\theta\left(F_{i}\right)=-2 n+r$ if $T_{i}=T\left(-\frac{1}{3}, \frac{1}{2} ;-4\right)$. Hence if $K=K_{1}=T\left(\frac{1}{3},-\frac{1}{2} ; 4\right) \cup_{\eta} T\left(\frac{1}{3},-\frac{1}{2} ; 4\right)$, then by Lemma 5.5 the boundary slope of $T$ is $[(2 n-r)+(2 n-r)] / n=3$. Similarly for the other two cases.

By Lemma 5.3, we have $|\partial F|=n=2 r$, and by Lemma 5.7 we have $r=2 s_{i}$, and hence $|\partial F|$ is a multiple of 4 .

\section{Toroidal surgery}

Let $(K, \delta)$ be one of the three pairs described in Theorem 1.1. In this section we show that there is a punctured torus $F$ in $E(K)$ with boundary slope $\delta$, and that the torus $\hat{F}$ obtained by capping off the boundary components of $F$ with meridional disks in the Dehn filling solid is indeed an essential torus in the surgered manifold $K(\delta)$.

Let $T=T\left(\frac{1}{3}, \frac{1}{2}\right)=T_{1} \cup T_{2}$, where $T_{1}=T\left(\frac{1}{3}\right)$ and $T_{2}=T\left(\frac{1}{2}\right)$.

Lemma 6.1. A special surface $Q$ in $E(T)$ is incompressible and $P$-incompressible.

Proof. By considering a component if necessary, we may assume that $Q$ is connected. By Lemma 3.3, each special disk has zero index, so by the Additivity Lemma 3.2 $Q$ also has zero index and hence is either an annulus disjoint from $U_{+}(T)$ or a disk intersecting $U_{+}(T)$ in two arcs. If $Q$ is a disk then it is automatically incompressible, and a $P$-compression will produce two disks $D_{i}$, each intersecting $U_{+}(T)$ at a single arc, which contradicts Lemma 3.4.

Now assume $Q$ is an annulus. Let $P^{\prime}=P_{+}\left(T_{1}\right)=P_{-}\left(T_{2}\right)$ be the twice-punctured disk cutting $E(T)$ into $E\left(T_{1}\right)$ and $E\left(T_{2}\right)$. By definition, $P^{\prime}$ cuts $Q$ into a set of special disks, each of which is an essential bigon in the sense that it intersects $P^{\prime}$ in two arcs, and an arc on the bigon with one endpoint on each of these arcs is not rel $\partial$ homotopic to an arc on $P^{\prime}$. Using this and the fact that $P^{\prime}$ is incompressible, one can easily show, by an innermost circle/outermost arc argument, that $Q$ is incompressible in $E(T)$. To show it is $P$-incompressible, one need only show that there is no $\partial$-compressing disk $D$ of $Q$ in $E(T)$ disjoint from $U_{+}(T)$. Suppose $\partial D=\alpha \cup \beta$, where $\alpha$ is an essential arc on $Q$ and $\beta \subset \partial E(T)-U_{+}(T)$. Since $P^{\prime}$ is incompressible, we may assume $D \cap P^{\prime}$ has no circle components; since $P^{\prime} \cap Q$ is a set of essential arcs on $Q, \alpha$ is isotopic to a component of $P^{\prime} \cap Q$, so by an isotopy of $D$ we may also assume that $\alpha \cap P^{\prime}=\varnothing$. Hence $D \cap P^{\prime}$ is a set of arcs with endpoints on $\beta$. Choose $D$ so that $\left|D \cap P^{\prime}\right|$ is minimal. If $D \cap P^{\prime}=\varnothing$, then one can use $D$ to $\partial$-compress a special disk to produce a pair of disks $D_{j}$ in some $E\left(T_{i}\right)$ with $\partial D_{j}$ the union of an essential arc on $P^{\prime}$ and another arc on $\partial E\left(T_{i}\right)-P^{\prime} \cup U_{+}(T)$, which will lead to a contradiction to Lemma 3.3. Now an 
outermost arc $\gamma$ cuts off a disk $D^{\prime}$ from $D$ which lies in one of the $E\left(T_{i}\right)$. Using Lemma 3.3, one can show that $\partial D^{\prime}$ is trivial on $\partial E\left(T_{i}\right)$, so it cuts off a 3-ball which can be used to reduce $\left|D \cap P^{\prime}\right|$, contradicting its minimality.

By definition, the knot $K$ is the union of two tangles $T_{1}, T_{2}$, each of which is either $T\left(\frac{1}{3},-\frac{1}{2} ; 4\right)$ or its mirror image $T\left(-\frac{1}{3}, \frac{1}{2} ;-4\right)$. Let $n=4, s=1$, and define $a_{k}$ by $a_{1}=a_{6}=1, a_{2}=a_{3}=3$, and $a_{4}=a_{5}=4$. One can check that these numbers satisfy the equations in Lemma 5.3. Since $T\left(\frac{1}{3},-\frac{1}{2} ; 4\right)$ is weakly equivalent to $T\left(\frac{1}{3}, \frac{1}{2}\right)$, by Lemma 5.3 there is a special surface $F_{i}$ in $E\left(T_{i}\right)$ that is the union of $a_{k}$ copies of special disks of type $(k)$ for $k=1, \ldots, 6$. By Lemma 5.4(iii), the curves $F_{i} \cap P(T)$ are represented by the train track in Figure 6(b), which splits to the one in Figure 7(5) because $r=2=2 s$. Similarly for $T_{i}=T\left(-\frac{1}{3}, \frac{1}{2} ;-4\right)$. The graph in Figure 7(5) is preserved by the gluing map $\eta: \partial B_{1} \rightarrow \partial B_{2}$, which by definition is a $(\pi / 2)$-rotation followed by a reflection along the vertical line. It follows that $F_{1} \cup_{\eta} F_{2}$ form a surface $F$ in $E(K)$.

Lemma 6.2. $F$ is a connected essential punctured torus in $E(K)$, with $\partial F$ consisting of 4 circles of slope $\delta$.

Proof. The boundary slope $\delta$ of $F$ is calculated in the proof of Proposition 5.8. In particular, $\delta$ is an integer in all three cases of $K$. Since $n=4$ in the construction, we see that $|\partial F|=4$.

Each $F_{i}$ consists of $\sum a_{i}=16$ disks, glued along $4 r+2(r+s)=14$ edges as shown in Figure 4(c)-(d), and hence $\chi\left(F_{i}\right)=16-14=2$. By Lemma 5.7, $F_{i} \cap P\left(T_{i}\right)$ is as shown in Figure 7(5), and hence it consists of $4 r=8$ arcs. Therefore $\chi(F)=\chi\left(F_{1}\right)+\chi\left(F_{2}\right)-8=-4$. Denote by $\hat{F}$ the surface obtained from $F$ by capping off each boundary component of $F$ with a disk. Since $|\partial F|=4$, we have $\chi(\hat{F})=0$. By Lemma 4.3, no component of $\hat{F}$ is a sphere. It follows that each component of $\hat{F}$ is a torus. In particular, no component of $F$ is an annulus.

By Lemma 6.1, each $F_{i}$ is incompressible and $P$-incompressible, and by Lemma 3.4, $P=P\left(T_{1}\right)=P\left(T_{2}\right)$ is also incompressible. By an innermost circle/outermost arc argument, one can thus show that $F=F_{1} \cup F_{2}$ is incompressible in $E(K)$. Since no component of $F$ is an annulus, this implies that $F$ is also $\partial$-incompressible, since otherwise two copies of a boundary compression disk and the annulus on $\partial N(K)$ bounded by two components of $\partial F$ would contain a compressing disk of $F$.

By Proposition 5.8, any essential punctured torus has at least 4 boundary components. Since $F$ has no closed component and $|\partial F|=4$, it follows that $F$ is connected.

Lemma 6.3. Let $M$ be a handlebody of genus 3, let $c_{1}, c_{2}$ be a pair of curves on $\partial M$, and let $M^{\prime}$ be the manifold obtained by attaching two 2-handles along $c_{1}$ and $c_{2}$. If $\partial M-c_{i}$ is compressible for $i=1,2$, and $\partial M-c_{1} \cup c_{2}$ is incompressible, then $\partial M^{\prime}$ is incompressible. 
Proof. This is a standard application of the handle addition lemma [Jaco 1984]. Denote by $M_{1}$ the manifold obtained from $M$ by attaching a 2-handle along $c_{1}$. By assumption, there is a compressing disk $D$ of $\partial M$ that is disjoint from $c_{1}$. If $D$ is separating, then one component $H$ of $M \mid D$ is a handlebody disjoint from $c_{1}$, so we may rechoose $D$ to be a nonseparating disk in $H$. Thus, after attaching a 2-handle to $M$ along $c_{1}, D$ is still a compressing disk of $\partial M_{1}$, so $\partial M_{1}$ is compressible. On the other hand, since $\partial M-c_{2}$ is compressible while $\left(\partial M-c_{2}\right)-c_{1}$ is incompressible, by the handle addition lemma applied to the pair $\left(M-c_{2}, c_{1}\right)$ we see that the surface $\partial M_{1}-c_{2}$ is incompressible in $M_{1}$. Now since $\partial M_{1}$ is compressible while $\partial M_{1}-c_{2}$ is incompressible, we may apply the handle addition lemma again to conclude that after attaching a 2-handle to $M_{1}$ along $c_{2}$, the boundary of the resulting manifold $M^{\prime}$ is incompressible.

Let $F_{0}$ be a separating surface in a 3-manifold $M$ with

$$
\partial F_{0}=c_{1} \cup \cdots \cup c_{n} \cup c_{n}^{\prime} \cup \cdots \cup c_{1}^{\prime},
$$

lying successively on a torus component $R$ of $\partial M$. Let $A_{1}$ be the component of $R \mid \partial F_{0}$ bounded by $c_{1} \cup c_{1}^{\prime}$, and let $A_{k}$ be the annulus on $R$ that is bounded by $c_{k} \cup c_{k}^{\prime}$ and contains $A_{1}$. Starting with $F_{0}$, one can construct a sequence of surfaces $F_{k}^{\prime}$ and $F_{k}$ by adding the annulus $A_{k}$ to $F_{k-1}$ to obtain $F_{k}^{\prime}$ and then pushing the $A_{k}$ part of $F_{k}^{\prime}$ off $R$ to obtain $F_{k}$. The surfaces $F_{k}$ are said to be obtained from $F$ by successively tubing through $A_{1}$. The following lemma is probably due to Gordon.

Lemma 6.4. Suppose $F_{0}$ is a connected separating incompressible surface in a 3manifold $M$ with $\partial F_{0}$ on a torus component $R$ of $\partial M$. Let $M_{0}^{\prime}, M_{0}^{\prime \prime}$ be the components of $M \mid F_{0}$, and let $A_{1}$ be an annulus component of $\partial M_{0}^{\prime}-\operatorname{Int} F_{0}$. If $F_{1}^{\prime}=F_{0} \cup A_{1}$ is incompressible in $M_{0}^{\prime}$, then the surfaces $F_{k}$ obtained by successively tubing $F$ through $A_{1}$ are all incompressible in $M$.

Proof. We use the above notation, and let $M_{k}^{\prime}, M_{k}^{\prime \prime}$ be the components of $M \mid F_{k}$. Note that $F_{k}$ are all connected and separating, and $A_{k+1}$ is a component of $\partial M_{k}^{\prime}-$ Int $F_{k}$ or $\partial M_{k}^{\prime \prime}-\operatorname{Int} F_{k}$. Hence, by induction, we need only show that $F_{1}$ is incompressible, and that if $n>1$ then $F_{2}^{\prime}$ is also incompressible in $M_{1}^{\prime \prime}$.

Since $F_{1}$ is obtained by pushing the $A_{1}$ part of $F_{1}^{\prime}=F_{0} \cup A_{1}$ into the interior of $M$, the component $M_{1}^{\prime}$ of $M \mid F_{1}$ contained in $M_{0}^{\prime}$ is homeomorphic to $M_{0}^{\prime}$, with $F_{1} \subset \partial M_{1}^{\prime}$ identified to $F_{0} \cup A_{1} \subset \partial M_{0}^{\prime}$, and so by assumption $F_{1}$ is incompressible in $M_{1}^{\prime}$. When $n=1$, the other component $M_{1}^{\prime \prime}$ is obtained by attaching a collar $R \times I$ to $M_{0}^{\prime \prime}$ along the annulus $A_{1}^{\prime}=R-\operatorname{Int} A_{1}$. It is clear that $A_{1}^{\prime}$ is incompressible in $M_{1}^{\prime \prime}$. If it is also $\partial$-incompressible, then an innermost circle/outermost arc argument shows that $F_{1}$ is incompressible in $M_{1}^{\prime \prime}$, and if it is $\partial$-compressible then $F_{0}$ must be an annulus that is parallel to $A_{1}^{\prime}$ after cutting off some possible summands, so $M_{1}^{\prime \prime}$ is essentially a product $R \times I$ and hence $F_{1}$ is also incompressible. When 
$n>1, M_{1}^{\prime \prime}$ is obtained by attaching $A_{2} \times I$ to $M_{0}^{\prime \prime}$ along the two annuli $P$ and $P^{\prime}$ bounded by $c_{1} \cup c_{2}$ and $c_{1}^{\prime} \cup c_{2}^{\prime}$. The incompressibility of $F_{0}$ implies that these annuli are incompressible. Also, there is no disk $D$ in $M_{1}^{\prime \prime}$ intersecting $P \cup P^{\prime}$ at a single essential arc, since otherwise the frontier of $N\left(D \cup P \cup P^{\prime}\right)$ would contain a compressing disk of $F_{0}$. One can now apply an innermost circle/outermost arc argument to show that both $F_{1}$ and $F_{2}^{\prime}$ are incompressible in $M_{1}^{\prime \prime}$.

Proof of Theorem 1.1. If $K$ is not a type II knot or if $\delta$ is not an integer slope, then by [Wu 1996b, Theorems 3.6 and 4.4], $K(\delta)$ is Haken and hyperbolic, so we assume that $K$ is a type II knot and $\delta$ is an integer slope. Write

$$
\left(S^{3}, K\right)=T_{1} \cup T_{2},
$$

with $T_{i}=T\left(p_{i} / q_{i}, \frac{1}{2}\right)$. By [Wu 1996b, Theorem 2.3], $E(K)$ contains an essential branched surface $\mathscr{B}$ that remains essential in $K(\delta)$, and hence by [Gabai and Oertel 1989] $K(\delta)$ is irreducible. Also, by the construction in the proof of [Wu $1996 \mathrm{~b}$, Theorem 2.3], the exterior of the $\mathscr{B}$ is the disjoint union of $E\left(T_{1}\right)$ and $E\left(T_{2}\right)$, with vertical surface $U_{+}\left(T_{i}\right)$ on $\partial E\left(T_{i}\right)$.

We claim that $E\left(T_{i}\right)$ is not an $I$-bundle with $U_{+}\left(T_{i}\right)$ as a vertical annulus. If this were false, then after attaching a 2-handle to $U_{+}\left(T_{i}\right)$, the resulting manifold $M_{i}$ would be an $I$-bundle over a closed surface, which must be a Klein bottle because $\partial M_{i}$ is a torus. Since $M_{i}$ is the exterior of a trefoil knot in $S^{3}$, this would imply that there is a Klein bottle embedded in $S^{3}$, which is absurd.

By [Brittenham 1998], if a small Seifert fiber space contains an essential lamination, then its exterior is an $I$-bundle; hence this implies that $K(\delta)$ cannot be a small Seifert fiber space. Therefore $K(\delta)$ is exceptional if and only if it is toroidal. By Proposition 5.8, $K(\delta)$ is toroidal only if $(K, \delta)$ is one of the three pairs listed, so we need only show that $K(\delta)$ is indeed a toroidal manifold for each of those pairs.

Let $F$ be the surface constructed before Lemma 6.2. By that lemma, $F$ is an essential punctured torus with $\partial F$ consisting of four circles of slope $\delta$ given in Theorem 1.1.

Let

$$
F_{i}=F \cap E\left(T_{i}\right), \quad P=P\left(T_{i}\right), \quad P_{i}=E\left(T_{i q}\right) \cap E\left(T_{i 2}\right) .
$$

The special disks $F \cap E\left(T_{i j}\right)$ in $E\left(T_{i j}\right)$ cut $E\left(T_{i j}\right)$ into a set of 3-balls $B_{k}$. The $\operatorname{arcs} P_{i} \cap F_{i}$ cut $P_{i}$ into a set of disks $D_{r}$, so the manifold $E\left(T_{i}\right) \mid F_{i}$ is obtained by gluing the $B_{k}$ along the $D_{r}$, and hence is a set of handlebodies $H_{k}$. Also, $F \cap P$ cuts $P$ into a set of disks $D_{r}^{\prime}$, so $E(K)$ is obtained from the $H_{k}$ by gluing along the $D_{r}^{\prime}$, and hence is also a set of handlebodies. Since $F$ is a punctured torus with four boundary components, it is separating in $E(K)$, so $E(K) \mid F$ has two components $M_{1}$ and $M_{2}$; and $\partial M_{i}$ is the union of $F$ with two annuli on $\partial N(K)$ and hence is a surface of genus 3. It follows that each $M_{i}$ is a handlebody of genus 3 . 
Let $A_{1}, A_{2}$ be the two annuli $\partial M_{i}-\operatorname{Int}(F)$, and let $c_{j}$ be the core of $A_{j}$. Then by Lemma 6.2 , the surface $\partial M_{i}-c_{1} \cup c_{2}$, which is homotopic to $F$ on $\partial M_{i}$, is incompressible. Note that $\partial M_{i}-c_{1}$ is homotopic to the surface $F \cup A_{2}$ obtained from $F$ by tubing along $A_{2}$. If this is incompressible, then by Lemma 6.4 the closed surface $F^{\prime}$ obtained from $F$ by successively tubing through $A_{2}$ is incompressible. From the construction, one can see that $F^{\prime}$ has coannular slope $\delta$ on $\partial N(K)$ in the sense that there is an incompressible annulus $A^{\prime}$ that has interior disjoint from $F^{\prime}$, with one boundary component on $F^{\prime}$ and the other on $\partial N(K)$ with slope $\delta$. It is easy to see that any embedded essential surface in an irreducible 3-manifold has at most one coannular slope on a torus boundary component, and hence there is no disk in $S^{3}$ with boundary on $F^{\prime}$ that intersects $K$ exactly once, so $F^{\prime}$ is $K$-essential in the sense of [Wu 1996b]. By Lemma 4.7 of that reference, there is no such closed surface in the exterior of a type II knot, which is a contradiction.

Let $\hat{M}_{1}, \hat{M}_{2}$ be the two components of $K(\delta) \mid \hat{F}$. Then $\hat{M}_{i}$ is obtained from $M_{i}$ by attaching two 2-handles along the curves $c_{1}, c_{2} \subset \partial M_{i}$. We have shown above that $M_{i}$ is a handlebody of genus $3, \partial M_{i}-c_{j}$ is compressible, and $\partial M_{i}-c_{1} \cup c_{2}$ is incompressible. Therefore by Lemma 6.3, the surface $\hat{F}=\partial \hat{M}_{i}$ is incompressible in $\hat{M}_{i}$. Since this is true for $i=1,2$, it follows that $\hat{F}$ is an incompressible torus in $K(\delta)$.

\section{References}

[Brittenham 1998] M. Brittenham, "Exceptional Seifert-fibered spaces and Dehn surgery on 2bridge knots”, Topology 37:3 (1998), 665-672. MR 99d:57011 Zbl 0912.57010

[Conway 1970] J. H. Conway, "An enumeration of knots and links, and some of their algebraic properties", pp. 329-358 in Computational Problems in Abstract Algebra (Oxford, 1967), Pergamon, Oxford, 1970. MR 41 \#2661 Zbl 0202.54703

[Eudave-Muñoz 1997] M. Eudave-Muñoz, "4-punctured tori in the exteriors of knots", J. Knot Theory Ramifications 6:5 (1997), 659-676. MR 98f:57022 Zbl 0887.57014

[Gabai 1986] D. Gabai, Genera of the arborescent links, Mem. Amer. Math. Soc. 339, American Mathematical Society, Providence, 1986. MR 87h:57010

[Gabai and Oertel 1989] D. Gabai and U. Oertel, "Essential laminations in 3-manifolds", Ann. of Math. (2) 130:1 (1989), 41-73. MR 90h:57012 Zbl 0685.57007

[Hatcher and Thurston 1985] A. Hatcher and W. Thurston, "Incompressible surfaces in 2-bridge knot complements”, Invent. Math. 79:2 (1985), 225-246. MR 86g:57003 Zbl 0602.57002

[Jaco 1984] W. Jaco, "Adding a 2-handle to a 3-manifold: an application to property R", Proc. Amer. Math. Soc. 92:2 (1984), 288-292. MR 86b:57006 Zbl 0564.57009

[Oertel 1984] U. Oertel, "Closed incompressible surfaces in complements of star links", Pacific J. Math. 111:1 (1984), 209-230. MR 85j:57008 Zbl 0549.57004

[Rolfsen 1990] D. Rolfsen, Knots and links, Math. Lecture Series 7, Publish or Perish, Houston, 1990. MR 95c:57018 Zbl 0854.57002

[Teragaito 2008] M. Teragaito, "Hyperbolic knots with three toroidal Dehn surgeries", J. Knot Theory Ramifications 17:9 (2008), 1051-1061. MR 2009g:57015 Zbl 05375316 
[Thurston 1979] W. P. Thurston, "The geometry and topology of three-manifolds", lecture notes, Princeton University, 1979, Available at http://library.msri.org/books/gt3m/.

[Wu 1996a] Y.-Q. Wu, "The classification of nonsimple algebraic tangles”, Math. Ann. 304:3 (1996), 457-480. MR 97b:57010 Zbl 0851.57019

[Wu 1996b] Y.-Q. Wu, "Dehn surgery on arborescent knots", J. Differential Geom. 43:1 (1996), 171-197. MR 97j:57013 Zbl 0851.57018

[Wu 1998] Y.-Q. Wu, "Sutured manifold hierarchies, essential laminations, and Dehn surgery", $J$. Differential Geom. 48:3 (1998), 407-437. MR 99h:57043 Zbl 0917.57015

Received March 8, 2010. Revised January 9, 2011.

YING-QING WU

DEPARTMENT OF MATHEMATICS

UNIVERSITY OF IOWA

14 MaCLEAN HALL

IOWA CITY, IA 52242-1419

UNITED STATES

wu@math.uiowa.edu 


\title{
PACIFIC JOURNAL OF MATHEMATICS
}

\author{
http://www.pjmath.org \\ Founded in 1951 by
}

E. F. Beckenbach (1906-1982) and F. Wolf (1904-1989)

\section{EDITORS}

V. S. Varadarajan (Managing Editor)

Department of Mathematics

University of California

Los Angeles, CA 90095-1555

pacific@math.ucla.edu

Vyjayanthi Chari

Department of Mathematics

University of California

Riverside, CA 92521-0135

chari@math.ucr.edu

\section{Robert Finn}

Department of Mathematics Stanford University

Stanford, CA 94305-2125

finn@math.stanford.edu

Kefeng Liu

Department of Mathematics

University of California

Los Angeles, CA 90095-1555

liu@math.ucla.edu
Darren Long

Department of Mathematics

University of California

Santa Barbara, CA 93106-3080

long@math.ucsb.edu

Jiang-Hua Lu

Department of Mathematics

The University of Hong Kong

Pokfulam Rd., Hong Kong jhlu@maths.hku.hk

Alexander Merkurjev

Department of Mathematics University of California

Los Angeles, CA 90095-1555 merkurev@math.ucla.edu
Sorin Popa

Department of Mathematics

University of California

Los Angeles, CA 90095-1555

popa@math.ucla.edu

Jie Qing

Department of Mathematics

University of California

Santa Cruz, CA 95064

qing@ cats.ucsc.edu

Jonathan Rogawski

Department of Mathematics

University of California

Los Angeles, CA 90095-1555

jonr@math.ucla.edu

\section{PRODUCTION}

pacific@math.berkeley.edu

Silvio Levy, Scientific Editor Matthew Cargo, Senior Production Editor

ACADEMIA SINICA, TAIPEI

CALIFORNIA INST. OF TECHNOLOGY

INST. DE MATEMÁTICA PURA E APLICADA

KEIO UNIVERSITY

MATH. SCIENCES RESEARCH INSTITUTE

NEW MEXICO STATE UNIV.

OREGON STATE UNIV.

\section{SUPPORTING INSTITUTIONS}

STANFORD UNIVERSITY
UNIV. OF BRITISH COLUMBIA
UNIV. OF CALIFORNIA, BERKELEY
UNIV. OF CALIFORNIA, DAVIS
UNIV. OF CALIFORNIA, LOS ANGELES
UNIV. OF CALIFORNIA, RIVERSIDE
UNIV. OF CALIFORNIA, SAN DIEGO
UNIV. OF CALIF., SANTA BARBARA

UNIV. OF CALIF., SANTA CRUZ

UNIV. OF MONTANA

UNIV. OF OREGON

UNIV. OF SOUTHERN CALIFORNIA

UNIV. OF UTAH

UNIV. OF WASHINGTON

WASHINGTON STATE UNIVERSITY

These supporting institutions contribute to the cost of publication of this Journal, but they are not owners or publishers and have no responsibility for its contents or policies.

See inside back cover or www.pjmath.org for submission instructions.

The subscription price for 2011 is US \$420/year for the electronic version, and \$485/year for print and electronic.

Subscriptions, requests for back issues from the last three years and changes of subscribers address should be sent to Pacific Journal of Mathematics, P.O. Box 4163, Berkeley, CA 94704-0163, U.S.A. Prior back issues are obtainable from Periodicals Service Company, 11 Main Street, Germantown, NY 12526-5635. The Pacific Journal of Mathematics is indexed by Mathematical Reviews, Zentralblatt MATH, PASCAL CNRS Index, Referativnyi Zhurnal, Current Mathematical Publications and the Science Citation Index.

The Pacific Journal of Mathematics (ISSN 0030-8730) at the University of California, c/o Department of Mathematics, 969 Evans Hall, Berkeley, CA 94720-3840, is published monthly except July and August. Periodical rate postage paid at Berkeley, CA 94704, and additional mailing offices. POSTMASTER: send address changes to Pacific Journal of Mathematics, P.O. Box 4163, Berkeley, CA 94704-0163.

PJM peer review and production are managed by EditFLOW ${ }^{\mathrm{TM}}$ from Mathematical Sciences Publishers.

PUBLISHED BY PACIFIC JOURNAL OF MATHEMATICS

at the University of California, Berkeley 94720-3840

A NON-PROFIT CORPORATION

Typeset in IATEX

Copyright $(2011$ by Pacific Journal of Mathematics 


\section{PACIFIC JOURNAL OF MATHEMATICS}

Volume $252 \quad$ No. $1 \quad$ July 2011

Some dynamic Wirtinger-type inequalities and their applications

RaVi P. Agarwal, Martin Bohner, Donal O'REgAn and SAMIR H.

SAKER

Splitting criteria for vector bundles on higher-dimensional varieties

PARSA BAKHTARY

Average Mahler's measure and $L_{p}$ norms of unimodular polynomials

KWOK-KWOng STEPHen CHOI and Michael J. MossinghofF

Tate resolutions and Weyman complexes

David A. CoX and EVgeny Materov

On pointed Hopf algebras over dihedral groups

FERNANDO FANTINO and GASTON ANDRÉS GARCIA

Integral topological quantum field theory for a one-holed torus

PATRicK M. Gilmer and Gregor Masbaum

Knot 4-genus and the rank of classes in $\boldsymbol{W}(\mathbb{Q}(t))$

CHARLES LIVINGSTON

Roots of Toeplitz operators on the Bergman space

ISSAM LOUHICHI and NAGISETTY V. RAO

Uniqueness of the foliation of constant mean curvature spheres in asymptotically 145 flat 3-manifolds

SHIGUANG MA

On the multiplicity of non-iterated periodic billiard trajectories

MARCo MAZZUCCHELLI

A remark on Einstein warped products

MiCHELE RIMOLDI

Exceptional Dehn surgery on large arborescent knots

YING-QING WU

Harnack estimates for the linear heat equation under the Ricci flow 